\title{
49th ANNUAL SASKATCHEWAN CHRISTMAS BIRD COUNT — 1990
}

\author{
Compiled by WAYNE C. HARRIS, Box 414, Raymore, Saskatchewan. SOA 3J0
}

The 1990 count was one of the coldest on record. Someone mentioned the words Christmas bird count on the 15 th (the first day of the count period) and Old Man Winter smiled and said "let there be winter" and there was - right through to the bitter end on 2 January (the last day of the count period). Many people waited in vain for the cold weather to break to do their counts, resulting in almost one third of the counts being done in the last two days! Only those who did their counts on the first two days escaped the bitterest cold.

\section{Weather and Coverage}

For details on just how cold it was (in case you don't remember) check Table 1. The coldest temperatures were logged by Endeavour and Squaw Rapids with $-42^{\circ} \mathrm{C}$ while Prince Albert(B) with an afternoon temperature $-35^{\circ} \mathrm{C}$ had the coldest high for the day. The coldest conditions were probably at Last Mountain Lake where a mid-day temperature of $-34^{\circ} \mathrm{C}$ was combined with a wind of $80 \mathrm{~km} / \mathrm{hr}$. Only a few counts on the first two days actually approached the zero mark. On an overall basis the average low was $-27^{\circ} \mathrm{C}$ and the average high was $-20^{\circ} \mathrm{C}$.

In spite of the cold weather, a total of 525 observers (see Count Areas and Participants following this introduction for names) on 79 counts tallied 87 species (plus one morph). Although all of these figures are above last year's totals, they are below the record numbers of 1987 and 1988. The total individuals seen on count day totalled 94,123. Coverage information is provided in Table 2 and shows that over $9200 \mathrm{~km}$ were driven in over 450 hours. Even with the cold weather an impressive $523 \mathrm{~km}$ were covered on foot in 284 hours. Not surprisingly almost as many hours (273) were spent in the comfort of homes watching feeders.

\section{The counts}

Of the 87 species recorded (81 on count day and six more during count period), 45 were seen on more than five counts and are presented in Table 3 . The most frequently occurring species were the Black-billed Magpie (74 of 79 counts), House Sparrow (68), Black-capped Chickadee (66) and Snow Bunting (55). The most abundant species was the Snow Bunting $(19,096$ total individuals) followed by House Sparrow $(17,762)$ and Mallard $(17,546)$. The greatest variety of species was reported from Saskatoon and Fort Walsh, both with 40. Only three other counts had 30 or more species; Raymore (39), Regina (37) and Squaw Rapids (30). Figure 1 shows the location of all counts.

\section{New species}

A single new species, Harlequin Duck, was added to the all time list. This bird had been present at Gardiner Dam for several weeks prior to the count and attracted great flocks(?) of birders all hoping to catch a glimpse of this male in full breeding plumage. This mountain breeding species is rare in Saskatchewan at any time but the Lake Diefenbaker area has accumulated a handful of records over the past few years including one down stream from Gardiner Dam near Outlook during the summer of 1990 (same individual?).

Although not a new species, the Black morph of the Rosy Finch (formerly a full species) was recorded for the first time in 
Saskatchewan at any time of the year. The most commonly occurring morph is the Gray-crowned but the Gray-headed has also been seen. The Black Rosy Finch normally occurs as close as southwestern Montana and to the best of my knowledge this record represents the first for Canada. This bird, found on the Big Muddy Lake count, was exceedingly tame, feeding as close as directly below the open window in the vehicle where the observers sat. An excellent description of the bird was submitted with the count.

\section{Rare species}

Table 4 presents the information on those species recorded on five or fewer counts. Some of the rarer species found this year include a Western Grebe at Qu'Appelle Dam (5th record), an American Black Duck at Gardiner Dam (3rd), a Turkey Vulture during count period at Edam (2nd), a Varied Thrush at Saskatoon (5th) and a count period Fox Sparrow at Raymore ( $3 \mathrm{rd}$ ). The second record of the Killdeer occurred on the same count, Govenlock, and at precisely the same location as the first record in 1982.

\section{Population trends and high counts}

Population levels were similar to last year for most species. There were however more Common Ravens south of the forest than usual while Snowy Owl numbers were generally down. Several species counts exceeded previous high counts (Table 6). Most impressive of these were the 10,000 Mallards at the Coronach power station on the Fife Lake count, the 274 Common Mergansers at Gardiner Dam and the 5445 Lapland Longspurs at Bromhead.

The following text and tables represents the compilation of this year's count. If you find any errors or ommissions please let me know.

\section{Count Areas And Participants}

(The names of compilers are in italics. The number of participants on each count are in parentheses following the list of names)

1. ABERNETHY-KATEPWA LAKE. Brian Bittner, Ron Bittner, Ron Hooper, Joy McKen, Norman McKen, Lorne Rowell, Keith Stevens, Wanda Stueck. (8)

2. ASSINIBOIA. Jack Burgeson, Cecil Hayward, David Landa, David Meagher, Wilf Prentice. (5)

3. BANGOR. Jean Hilton, Minnie Hughes, Sheila Popowich. (3)

4. BETHUNE. Douglas \& Vera Laing. (2)

5. BIGGAR. Roger Assailly, Guy Wapple, Marguerite Wapple, Robert Wapple. (4)

6. BIG MUDDY LAKE. Martin Bailey, Keith Barr, Carol Bjorklund, Paul Chytyk, Bob Luterbach, Milow Worel. (6)

7. BIG MUDDY VALLEY (south of Bengough). Daryl Johannesen. (1)

8. BIG RIVER. Glenn Honig. (1)

9. BIRCH HILLS. MoeMareschal, Don Weidl. (2)

10. BROADVIEW. Dave Chaskavich. (1)

11. BROMHEAD. Martin Bailey, Carol Bjorklund. (2)

12. CANDLE LAKE. Burke Korol, Arvid Pederson, Guy Wapple, Robert Wapple. (4)

13. CARRAGANA. Marg \& Stan Back, David Chapman, Herman Duerksen, Bill Howse, Glen Ormsby. (6)

14. CROOKED LAKE(A). Don Weidl. (1)

15. CROOKED LAKE(B). Bill Livesay, Mayta Livesay, Dorothy Skene, Ed Skene. (4)

16. DEVIL'S LAKE. Thomas Harper. (1)

17. DILKE. Margaret Belcher, Ken McArton. (2)

18. DOUGLAS PROVINCIAL PARK. Bob Plaster. (1)

19. DUVAL. George Herber, Merv Hey, Susan McAdam, lain Richardson, Lloyd Saul. (5) 
20. EASTEND. Henri Lebastard. (1)

21. EDAM. Doris \& Jim Forsyth, Dale \& Theresa Hamilton, Ernest \& Wilma Hardcastle, Owen Jones, Marg \& Tom Terpstra. (9)

22. ENDEAVOUR. Norman Harris. (1)

23. ESTEVAN. Don Weidl. (1)

24. FIFE LAKE. Martin Meyers, Robert Rafuse. (2)

25. FORT QU'APPELLE. Phyllis Bordass, William Bordass, Ethel Cockwill, Doreen Harman, Barbara Hooper, Ron Hooper, Alice Laing, Lois Lamontagne, Maurice Lindgren, Jack Lowe, Don McDougall, Mary Morris, Allan Mlazgar, Paul Paquin, Laurence Robillard, Doreen Rowell, Lorne Rowell, Ron Simmonds, Keith Stephens, Lloyd Talbot. (20)

26. FORT WALSH. Keith Barr, Bob \& Dixie Black, Anne Harris, Valeri Harris, Wayne Harris, Burke Korol, Robert Kreba, Sheila Lamont, Ron Myers, Brian Olson, Wilkes Parsonage, Guy Wapple, Robert Wapple, Milow Worel. (15)

27. GARDINER DAM. Martin Bailey, Carol Bjorklund, Paul Coutu, Anne Harris, Valeri Harris, Wayne Harris, Burke Korol, Sheila Lamont, Menno Nickel, Brian Olson, Frank Roy, Guy Wapple, Robert Wapple, Michael Williams. (14)

28. GOOD SPIRIT LAKE. Bill Anaka, Joyce Anaka, Julia Wiwchar. (3)

29. GOVENLOCK. Keith Barr, Anne Harris, Valeri Harris, Wayne Harris, Burke Korol, Robert Kreba, Sheila Lamont, Ron Myers, Brian Oison, Guy Wapple, Robert Wapple, Milow Worel. (12)

30. GRAND CENTRE-PIERCELAND. Frank Roy, Heidi Roy, Jessica Roy, Robin Roy. (4)

31. GRASSLANDS NATIONAL PARK. Paul Chytyk, Wayne Harris, Sheila Lamont. (3)

32. HUDSON BAY. Pat Beaulieu. (1)

33. HUMBOLDT. Ed Brockmeyer, Mike Volk. (2)

34. INDIAN HEAD. Margo Beaulieu, Vic Beaulieu, Bernard Bosgoed, Gordon
Howe, John Kort, Bruce Neill, Fred Skinner, Gordon Willerth. (8)

35. KAMSACK. Mrs. Bernhard, Mable Bucuek, Lynnde Dewores, Fred Erhart, Doris \& Bert Franklin, Anita Klochko, Rezansoff family, Isabel Ritchie, John Solamon, Elenor Sookocheff, Helen Tomochko, Elona Zeiben. (15)

36. KELVINGTON. Marguerite Sloan. (1)

37. KENASTON. Doug Beckie, Lawrence Beckie. (2)

38. KINDERSLEY(A). Beth Dales, Jean Harris. (2)

39. KINDERSLEY(B). Gerry Essar, Michael Essar. (2)

40. KUTAWAGAN LAKE. Wayne Harris, Susan McAdam. (2)

41. LA RONGE. Jim Paul, Lorieann Paul. (2)

42. LAST MOUNTAIN LAKE WILDLIFE MANAGEMENT UNIT. Wayne Harris, Sheila Lamont. (2)

43. LEADER. Daisy Meyers. (1)

44. LIVELONG. Sarah Pavka, Vicky Tollefson. (2)

45. LOVE-TORCH RIVER. Dot Bennett, Ed Bennett, Bert Dalziel, Duke Dalziel, Kari Dalziel, Joan Dalziel, Sara Dalziel, Mildred Long, Bill Matthews, Lynn Matthews. (10)

46. LUSELAND. Estelle Finley, Kim Finley, Bill Frey, Joe Rozlein. (4)

47. MAIDSTONE BRIDGE. Anne Harris, Valeri Harris, Wayne Harris, Dan Lamont, Sheila Lamont, Tom Lamont. (6)

48. MARSDEN. Eileen Graham, Candi Schopper, Lois Wooding (non-participating compiler). (2)

49. MELVILLE. Donna Alberts, Marion MacLean, Robert Wotherspoon, Ross Wotherspoon. (4)

50. MISSINIPE. Shirley Glass. (1)

51. MOOSE JAW. Mac, Merran, Pat \& Riel Aldred, Edith Bell, Vi Belt, Helen 
Brunsdon, Muriel Dormer, Joan Finn, Doug \& Pat Francis, Allan, Edward, Hartley, Kenneth, Lawrence \& Tristan Fredeen, Florine Hales, Kerry Hanley, Cy Knight, Leith Knight, Ann Kovlaske, Kay Kreuger, Connie \& Hugh Mclntyre, Flo Noble, Wilma Pickering, Henry Van Dorland, Sheina Wait, Ed \& Grace Walker, Bev Wenzel. (32)

52. NEELY LAKE. Edwin Billeter, Donald Hooper, Maureen Johnson. (3)

53. PIKE L.AKE. Carol Blenkin, Sandra Flood, Mary Gilliland, Bernie Gollop, Mike Gollop, Frank Roy, Jacob Stegeman, Jim Wedgwood, Michael Williams, Lois Wooding. (1)

54. PORCUPINE PLAIN. Les Baker, Agnus Hodge, Glen Hodge, Donald Hooper, Margaret Hooper, Brian Larwood, Keith Larwood, Pat Larwood. (8)

\section{PRINCE ALBERT(A). Elsie \& FrankMorton. (2)}

56. PRINCE ALBERT(B). Adam Beck, Carol Beck, Ron Beck, John Burt, Pam Burt, Evelyn Chapais, Carman Dodge, Keith Dodge, Joe Graumans, Hamilton Greenwood, Cora Pond, Ted Snow. (12)

57. PRINCE ALBERT NATIONAL PARK. Marilyn Anions, Roger Baird, Mark Boyd, Susan Carr, Chris Clinton, Eileen Clinton, Andree Galbraith, Paul Galbraith, Jim Helm, Doug Hodgins, Arnold Janzen, Kelly Jennett, Gary Kolar, Susan Kolar, Erin Kurtz, Bob Luterbach, Bradley Muir, Bill Prettie, Cory Rolles, Frank Roy, Jennifer Skage, Kristen Skage, Sharon Skage, Jim Slimmon, Merv Syroteuk, Jenny Tibbs, Bob Turnbull, Jim Wedgwood, Jeff Weir, Dana Wiberg. (30)

58. QU'APPELLE DAM. Stan Shaddick, Michael Williams. (2)

59. RAYMORE. Anne Harris, Valeri Harris, Wayne Harris, Sheila Lamont. (4)

60. REGINA. Martin Bailey, Donna Barclay, Keith Barr, Margaret Belcher, Carol Bjorklund, Paul Chytyk, Lyn Crowley, Stu Dechka, Bob Decker, Marilyn Decker, Peter Heron, Trevor Herriot, Paul James, Bill Jones, Bob Kreba, Linda Langenbacher,
Wolfgang Langenbacher, Christine MacDonald, John McDonald, Ron Myers, John Nelson, Marian Peddle, Ron Peddle, John Pollock, Brian Rainey, Dean Rickert, Miriam Rickert, Tom Riffel, Keating Smith, Marilyn Smith, Frank Switzer, Ian Switzer, Raymond Tervo, Reino Tervo, Milow Worel. (35)

61. ROUND LAKE. Doug Francis, Pat Francis, Joe Grimeau, Laura Grimeau. (4)

62. SASKATOON. Norma Allen, jill Anweiler, Juhachi Asai, Kengo Asai, Mika Asai, Rika Asai, Sumiko Asai, Bob Besant, Garth Besant, Joyce Besant, Barry Betker, Carol Blenkin, Earl Blewett, Eveline Boudreau, Paul Coutu, Tony Diamond, Ed Driver, Anne Dzus, Jan Earl, Steve Earl, Helen Elliott, Martin Gerrard, Silvia Gerrard, Colleen Gerwing, Mary Gilliland, Bob Godwin, Bernie Gollop, Madeleine Gollop, Fred Goodliffe, Bob Green, Carol Green, Jerry Haight, Jo Haight, Bruce Hanbidge, John Hanbidge, Ramona Harms, Mary Houston, Stuart Houston, Brian Johns, Richard Kerbes, Janice Laliberte, Harv Lane, Ken Lumbis, Edna McCawley, Don McRobbie, Jo McRobbie, Kathy Meeres, Meewasin Beaver Creek staff and cooperators (3), Cathryn Miller, David Miller, Quentin Miller, Arlene Moe, Willis Moe, Menno Nickel, Delores Norman, Brian Olson, Valerie Olson, Jim Pepper, Janet Pivnick, Ken Pivnick, Jim Slimmon, Alan Smith, Henry Tabel, Phil Taylor, David Thorpe, Garth Van Derkamp, Bernadette Vangool, Koren Vangool, Heather Wagg, Jim Wedgwood, Michael Williams, Jim Wood, Lois Wooding, Dan Zazalenchuk. (76)

63. SCOTT. Guy Wapple, Sandra Wapple. (2)

64. SKULL CREEK. Bob \& Moreen Bell, Jim Bennetto, Bob Eccleston, Marj Mann Jr., Larry Peterson, Pat \& Pete Robertshaw, Michelle \& Ray Shuler. (10)

65. SNOWDEN. Ed Hagel, Irene Hagel, Karen Hagel, Iain Richardson, Lloyd Saul. (5)

66. SOMME. David Black, Evelyn Black, Ron Butterfield, Donald Hooper, Margaret Hooper. (5) 
67. SPALDING. Bill Spizawka, Velma Spizawka. (2)

68. SPINNEY HILL. Ed Driver, Phil Taylor. (2)

69. SPRING VALLEY. Allan Bogdan, Bryan Bogdan, Flossie Bogdan, Larry Bogdan, Nick Bogdan, Dean Goian. (6)

70. SQUAW RAPIDS. Wayne Harris, Burke Korol, Sheila Lamont, Guy Wapple, Robert Wapple. (5)

\section{SWIFT CURRENT. Ron Jensen. (1)}

72. TISDALE. Joyce Mohr, Brian Yelland, Llyn Yelland. (3)

\section{TURTLE LAKE. E. Robinson, V. Robinson. (2)}

74. WEYBURN. E. Baird, L. Belanger, Leo Belanger, Ray Belanger, Greg Bobbitt, R. Churchill, R. Douglas, N. Flaten, F. Garner, Dick Gutfriend, D. Hannah, D. Herber- holz, G. Kurtz, B. Layh, P. Layh, Nick Postey, H. Stairmand, Stewart Stairmand, J. Weisaerber, R. Weisaerber. (20)

75. WHITE BEAR. Gary Jordheim, Sig Jordheim. (2)

76. WHITE BEAR LAKE. Ray Belanger, Greg Bobbitt, Sheila Burland, Dick Gutfriend, Veronica Pasterfield, Stewart Stairmand. (6)

77. WHITEBEECH. John Kereliuk, Ed Terlesky, Ida Wotherspoon, Lindsay Wotherspoon. (4)

78. WHITEWOOD. John Pollock. (1)

79. YORKTON. Loraine Arnold, Joyce Bains, Derek Eftoda, Jim Eftoda, Lea Herauf, Warren Hjertaas, Sharon Kozak, Lorne Lepp, Marilyn Lepp, Dorothy Skene, Ed Skene, Alice Turberfield, Beth Turberfield, Gordon Turberfield, Harold Wilkinson. (15)

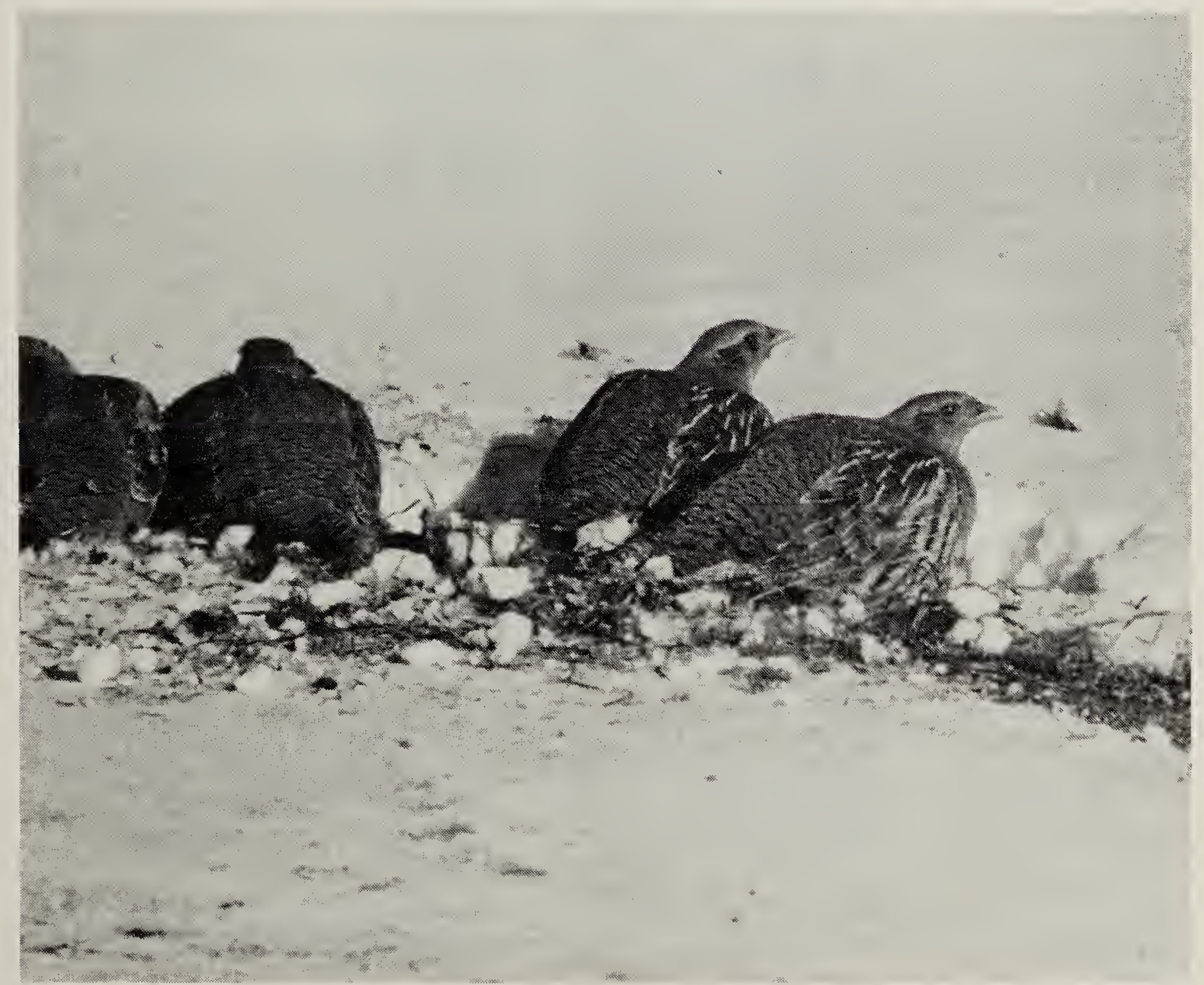


Table 1. COUNT WEATHER CONDITIONS $\left(\mathrm{T}^{\circ} \mathrm{C}\right.$, Wind $\mathrm{Kmph}$, Snow $\left.\mathrm{Cm}\right)$

\begin{tabular}{|c|c|c|c|c|c|c|c|c|}
\hline COUNT NAME & $\begin{array}{l}\text { Min } \\
T^{\circ} \mathrm{C}\end{array}$ & $\begin{array}{l}\text { Max. } \\
T^{\circ} \mathrm{C}\end{array}$ & $\begin{array}{l}\text { Min. } \\
\text { Wind }\end{array}$ & $\begin{array}{l}\text { Max. } \\
\text { Wind }\end{array}$ & $\begin{array}{l}\text { Min. } \\
\text { Snow }\end{array}$ & $\begin{array}{l}\text { Max. } \\
\text { Snow }\end{array}$ & Sky A.M. & Sky P.M. \\
\hline $\begin{array}{l}\text { ABERNETHY-KATEPWA LAKE } \\
\text { ASSINIBOIA } \\
\text { BANGOR } \\
\text { BETHUNE } \\
\text { BIGGAR }\end{array}$ & $\begin{array}{l}-38 \\
-20 \\
-30 \\
-35 \\
-32 \\
\end{array}$ & $\begin{array}{r}-34 \\
+3 \\
-27 \\
-25 \\
-27 \\
\end{array}$ & \begin{tabular}{|r|}
0 \\
0 \\
20 \\
0 \\
10 \\
\end{tabular} & $\begin{array}{r}5 \\
15 \\
25 \\
13 \\
20 \\
\end{array}$ & $\begin{array}{r}20 \\
10 \\
0 \\
\end{array}$ & $\begin{array}{l}25 \\
10 \\
30 \\
15 \\
20 \\
\end{array}$ & $\begin{array}{l}\text { overcast } \\
\text { mostly clear } \\
\text { mostly cloudy }\end{array}$ & $\begin{array}{l}\text { partly cloudy } \\
\text { clear } \\
\text { mostly clear } \\
\text { clear } \\
\text { mostly cloudy }\end{array}$ \\
\hline BIC MUDDY LAKE & -20 & -10 & 5 & 10 & 0 & 10 & clear & clear \\
\hline BIG MUDDY VALLEY (s. of Bengough) & -25 & -20 & 15 & 20 & 2 & 30 & partly cloudy & partly cloudy \\
\hline BIG RIVER & -37 & -25 & 10 & 20 & 30 & 35 & mostly clear & mostly clear \\
\hline BIRCH HILL5 & -31 & -29 & 0 & 5 & 6 & 30 & mostly clear & mostly clear \\
\hline BROADVIEW & -24 & -20 & 30 & 35 & 10 & 20 & clear & clear \\
\hline BROMHEAD & -34 & $-\overline{24}$ & 8 & $\overline{16}$ & 5 & 15 & mostly clear & mostly clear \\
\hline CANDLE LAKE & -30 & -25 & 5 & 40 & 30 & 50 & clear & clear \\
\hline CARRAGANA & -35 & -22 & 10 & 12 & 31 & 27 & partly cloudy & mostly clear \\
\hline CROOKED LAKE(A) & -18 & -17 & 15 & 20 & 15 & 25 & mostly cloudy & mostly clear \\
\hline CROOKED LAKE(B) & -37 & -30 & 30 & 50 & 20 & 45 & & mostly clear \\
\hline DEVIL'5 LAKE & -31 & -28 & 15 & 20 & 7 & 8 & overcast & overcast, light snow \\
\hline DILKE & -5 & -6 & 15 & 60 & 0 & 15 & overcast, light snow & \\
\hline DOUGLAS PROVINCIAL PARK & -30 & -25 & 10 & 15 & 15 & 25 & & \\
\hline DUVAL & -30 & -38 & 5 & 29 & 6 & 43 & mostly clear & clear \\
\hline EA5TEND & -30 & -24 & 0 & 10 & & & & mostly clear \\
\hline EDAM & & -21 & & & & & & \\
\hline ENDEAVOUR & -42 & -32 & 0 & 0 & 36 & 38 & clear & clear \\
\hline ESTEVAN & -38 & -26 & 15 & 20 & 10 & 15 & clear & clear \\
\hline FIFE LAKE & -25 & -20 & 20 & 35 & 0 & 30 & partly cloudy & mostly clear \\
\hline FORT QU'APPELLE & -18 & -11 & 0 & 0 & 8 & 8 & mostly clear & clear \\
\hline FORT WALSH & -10 & -2 & 0 & 60 & 0 & 25 & clear & clear \\
\hline GARDINER DAM & -23 & -10 & 10 & 30 & 5 & 20 & mostly cloudy & mostly cloudy \\
\hline GOOD SPIRIT LAKE & -28 & -25 & 20 & 30 & 10 & 22 & clear & clear \\
\hline GOVENLOCK & -8 & -4 & 0 & 15 & 0 & 2 & partly cloudy & clear \\
\hline GRAND CENTRE-PIERCELAND & -26 & -16 & 6 & 15 & 20 & 30 & overcast & overcast \\
\hline GRASSLANDS NATIONAL PARK & -18 & -12 & 10 & 50 & 0 & 10 & clear & partly cloudy \\
\hline HUD5ON BAY & -30 & -25 & 15 & 20 & 25 & 30 & & \\
\hline HUMBOLDT & -27 & -25 & 15 & 30 & 6 & 10 & overcast, light snow & overcast \\
\hline INDIAN HEAD & -24 & -22 & 30 & 15 & 5 & 20 & & partly cloudy \\
\hline KAM5ACK & -30 & -34 & & & & & mostly clear & mostly clear \\
\hline KELVINGTON & -22 & -10 & & & 20 & 25 & clear & \\
\hline KENASTON & -25 & -11 & 0 & 10 & 10 & 15 & overcast & overcasl \\
\hline KINDERSLEY(A) & & & & & 2 & 5 & clear & clear \\
\hline KINDER5LEY (B) & -30 & -28 & 15 & 21 & 7 & 30 & partly cloudy & mosily cloudy \\
\hline KUTAWAGAN LAKE & -32 & -23 & 10 & 40 & 1 & 20 & overcast & overcast \\
\hline LARONGE & -34 & -19 & 0 & 14 & 25 & 40 & mostly clear & partly cloudy \\
\hline LA5T MOUNTAIN LAKE W.M.U. & -35 & -34 & 30 & 80 & 0 & 15 & clear & clear, blowing snow \\
\hline LEADER & -28 & -22 & 0 & 0 & & & mostly clear & overcast, light snow \\
\hline LIVELONG & -32 & -25 & 15 & 20 & 20 & 25 & mostly clear & partly cloudy \\
\hline LOVE-TORCH RIVER & -35 & -25 & 0 & 10 & 45 & 60 & clear & clear \\
\hline LUSELAND & -24 & -18 & 15 & 35 & 2 & 90 & clear & clear \\
\hline MAIDSTONE BRIDGE & -31 & -23 & 2 & 30 & 0 & 30 & mostly clear & mostly clear \\
\hline MARSDEN & & & & & & & & \\
\hline MELVILLE & -38 & -28 & 25 & 40 & 0 & 23 & partly cloudy & mostly clear \\
\hline MI55INIPE & -28 & -20 & 0 & 5 & 25 & 30 & overcast & overcast \\
\hline MOO5E IAW & -23 & -14 & 6 & 8 & 12 & 25 & mostly cloudy & mosily cloudy \\
\hline NEELY LAKE & -35 & -32 & 15 & 20 & 15 & 20 & clear & clear \\
\hline PIKE LAKE & -38 & -31 & 13 & 20 & & 25 & mostly clear & mostly clear \\
\hline $\begin{array}{l}\text { PORCUPINE PLAIN } \\
\text { PRINCE ALBERT(A) }\end{array}$ & -21 & -9 & 0 & 0 & 80 & 100 & clear & clear \\
\hline PRINCE ALBERT(B) & $-\overline{39}$ & -35 & 0 & 0 & 10 & 15 & partly cloudy & partly cloudy \\
\hline PRINCE ALBERT NATIONAL PARK & -21 & -15 & 0 & 10 & 28 & 37 & mostly cloudy & mostly clear \\
\hline QU'APPELLE DAM & -10 & -5 & 10 & 30 & 1 & 30 & mostly clear & mostly clear \\
\hline RAYMORE & -38 & -29 & 0 & 10 & 2 & 25 & clear & clear \\
\hline REGINA & -35 & -18 & 0 & 10 & 5 & 15 & partly cloudy & partly cloudy \\
\hline ROUND LAKE & -25 & -18 & 15 & 25 & 3 & 15 & & \\
\hline SASKATOON & -28 & -15 & 6 & 13 & 16 & 20 & mostly cloucly & mostly cloudy \\
\hline $5 \mathrm{COT}$ & -32 & -25 & 10 & 20 & 0 & 15 & clear & clear \\
\hline SKULL CREEK & -10 & -3 & 25 & 30 & 30 & 36 & mostly clear & mostlv clear \\
\hline 5NOWDEN & -22 & -18 & 0 & 20 & 4 & 50 & clear & partly cloudy \\
\hline $5 \mathrm{OMME}$ & -22 & -12 & 9 & 18 & 110 & 150 & partly cloudy & mostly cloudy \\
\hline 5PALDING & -28 & -24 & 35 & 45 & 12 & 16 & clear & clear \\
\hline 5PINNEY HILL & -25 & -20 & 13 & 25 & 0 & 25 & clear & mostly clear \\
\hline SPRING VALLEY & -23 & $-1+$ & 8 & 65 & & 10 & overcast, light snow & overcast, light snow \\
\hline 5QUAW RAPID5 & -42 & -26 & 10 & 30 & 25 & 30 & clear & mostly clear \\
\hline 5WIFT CURRENT & -18 & & & & & & overcast & mostly cloudy \\
\hline TISDALE & -20 & -30 & 15 & 20 & & 15 & clear & mostly clear \\
\hline TURTLE LAKE & -30 & -25 & 2 & 10 & & 30 & & \\
\hline WEYBURN & -20 & -18 & 5 & 15 & 2 & 6 & partly cloudv & partly cloudy \\
\hline WHITE BEAR & -25 & -16 & 0 & 1 & 5 & 120 & mostly clear & partly cloudy \\
\hline WHITE BEAR LAKE & -27 & -23 & 22 & 30 & 4 & 15 & mostly cloudy & mostly cloudy \\
\hline WHITEBEECH & -23 & -20 & 3 & 5 & 24 & 28 & & clear \\
\hline WHITEWOOD & -20 & -15 & 5 & 15 & 0 & 20 & mostly clear & mostly clear \\
\hline YORKTON & -28 & -20 & 0 & 10 & & & clear & clear \\
\hline
\end{tabular}


1. ABERNETHY-KATEPWA LAKE

2. ASSINIBOIA

3. BANGOR

4. BETHUNE

5. BIGGAR

6. BIG MUDDY LAKE

7. BIG MUDDY VALLEY

8. BIG RIVER

9. BIRCH HILLS

10. BROADVIEW

11. BROMHEAD

12. CANDLE LAKE

13. CARRAGANA

14. CROOKED LAKE(A)

15. CROOKED LAKE(B)

16. DEVIL'S LAKE

17. DILKE

18. DOUGLAS PROVINCIAL PARK

19. DUVAL

20. EASTEND

21. EDAM

22. ENDEAVOUR

23. ESTEVAN

24. FIFE LAKE

25. FORT QU'APPELLE

26. FORT WALSH
27. GARDINER DAM

28. GOOD SPIRIT LAKE

29. GOVENLOCK

30. GRAND CENTRE-PIERCELAND

31. GRASSLANDS NATIONAL PARK

32. HUDSON BAY

33. HUMBOLDT

34. INDIAN HEAD

35. KAMSACK

36. KELVINGTON

37. KENASTON

38. $\operatorname{KINDERSLEY(A)}$

39. KINDERSLEY(B)

40. KUTAWAGAN LAKE

41. LA RONGE

42. LAST MOUNTAIN LAKE W.M.U. 69. SPRING VALLEY

43. LEADER

44. LIVELONG

45. LOVE-TORCH RIVER

46. LUSELAND

47. MAIDSTONE BRIDGE

48. MARSDEN

49. MELVILLE

50. MISSINIPE

51. MOOSE JAW

52. NEELY LAKE

53. PIKE LAKE
54. PORCUPINE PLAIN

55. PRINCE ALBERT(A)

56. PRINCE ALBERT(B)

57. PRINCE ALBERT NATIONAL PARK

60. REGINA

61. ROUND LAKE

62. SASKATOON

63. SCOTT

64. SKULL CREEK

65. SNOWDEN

66. SOMME

67. SPALDING

70. SQUAW RAPIDS

71. SWIFT CURRENT

72. TISDALE

73. TURTLE LAKE

74. WEYBURN

75. WHITE BEAR

76. WHITE BEAR LAKE

77. WHITEBEECH

78. WHITEWOOD

79. YORKTON

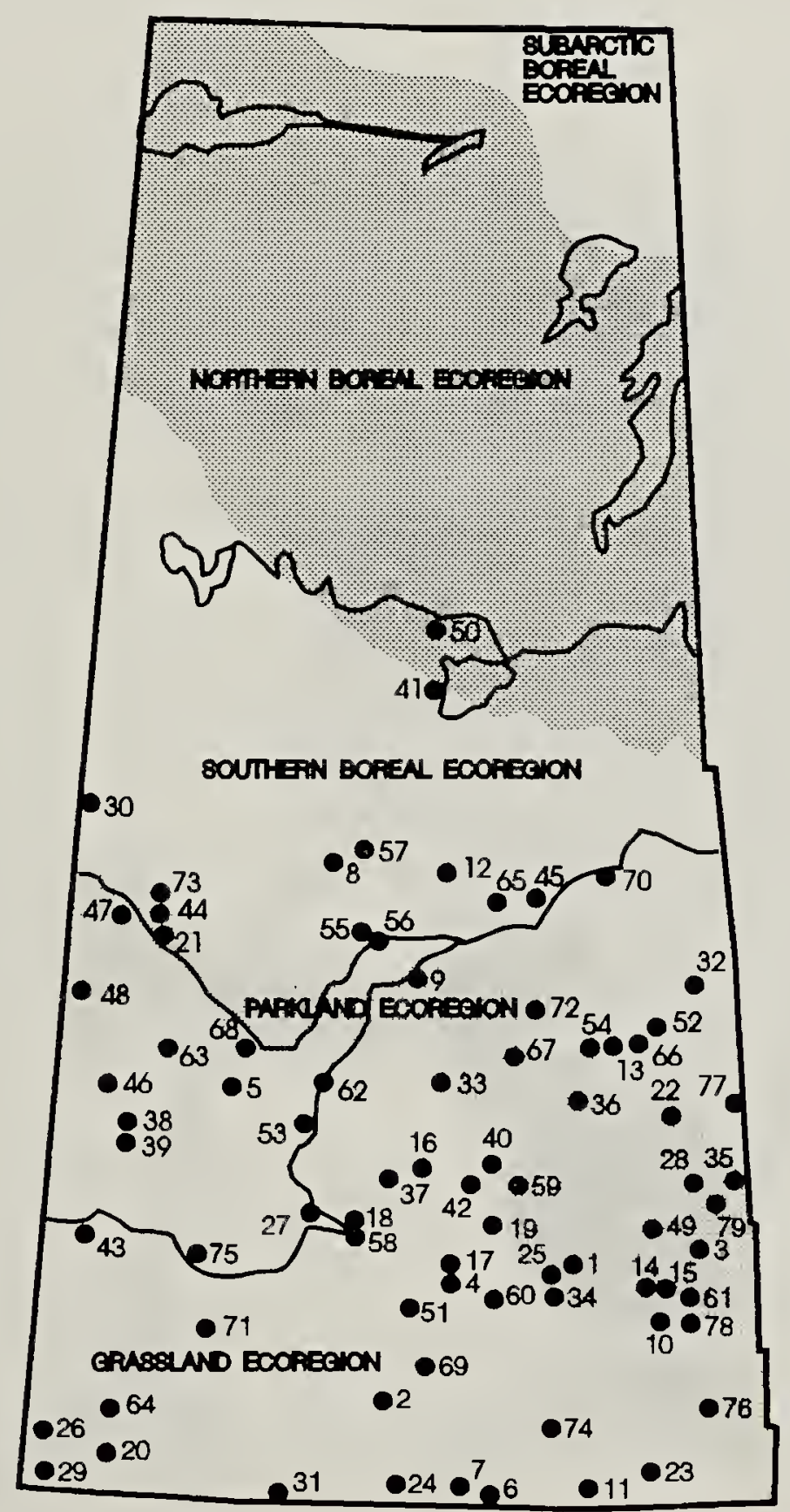

Figure 1. Location of 1990 counts. 
Table 2. COUNT COVERAGE (* indicates counts which conform to the North American standard of all day counts covering a 15 mile diameter circle)

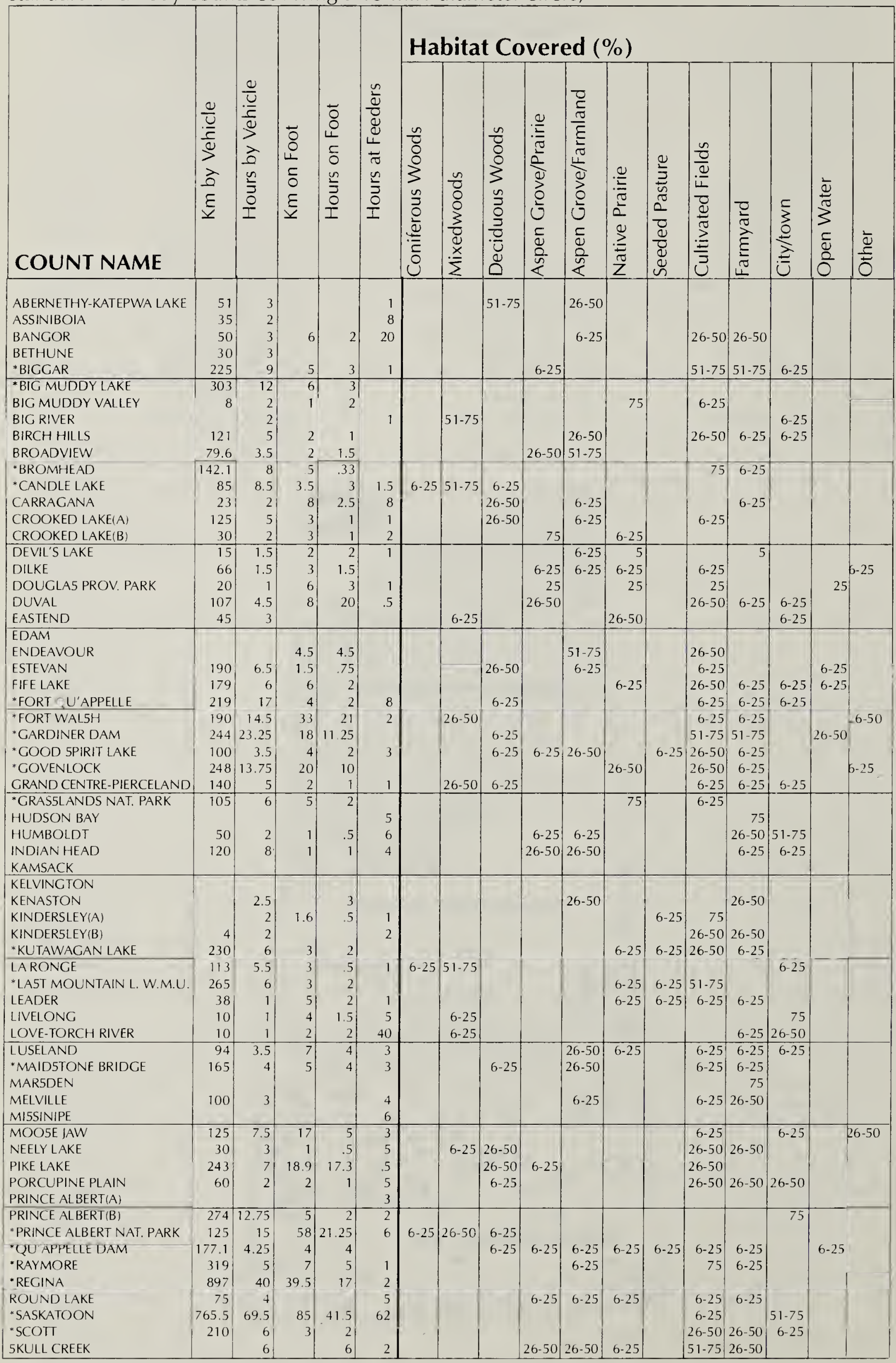


Table 2. COUNT COVERAGE (* indicates counts which conform to the North American standard of all day counts covering a 15 mile diameter circle)

\begin{tabular}{|c|c|c|c|c|c|c|c|c|c|c|c|c|c|c|c|c|c|}
\hline \multirow[b]{2}{*}{ COUNT NAME } & \multirow[b]{2}{*}{$\begin{array}{l}\frac{0}{v} \\
\frac{0}{2} \\
\frac{\partial}{2} \\
\frac{\partial}{E} \\
\frac{E}{y}\end{array}$} & \multirow[b]{2}{*}{$\begin{array}{l}\frac{0}{\frac{u}{2}} \\
\frac{0}{2} \\
\sum^{2} \\
\frac{2}{0} \\
\frac{n}{3} \\
\frac{0}{1} \\
1\end{array}$} & \multirow[b]{2}{*}{$\begin{array}{l}5 \\
0 \\
1 \\
\overline{0} \\
\overline{5} \\
\bar{y}\end{array}$} & \multirow[b]{2}{*}{ 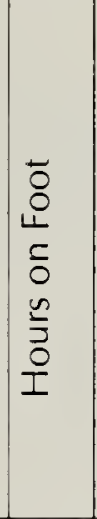 } & \multirow[b]{2}{*}{ 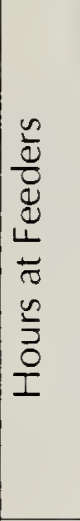 } & \multicolumn{12}{|c|}{ Habitat Covered ( $\%$ ) } \\
\hline & & & & & & 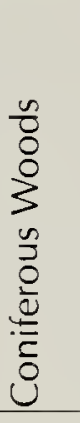 & 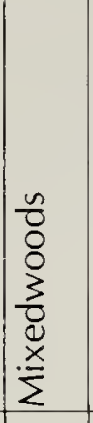 & $\begin{array}{l}\frac{n}{0} \\
8 \\
0 \\
3 \\
n \\
0 \\
0 \\
\frac{3}{0} \\
\frac{0}{U} \\
0 \\
0 \\
0\end{array}$ & 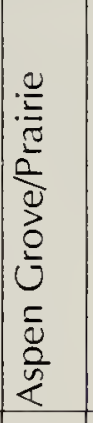 & 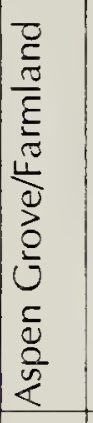 & 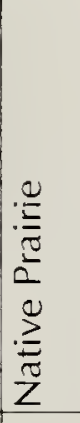 & 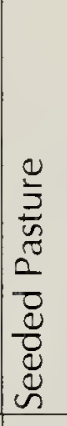 & $\begin{array}{l}\frac{n}{0} \\
\frac{0}{2} \\
\frac{0}{0} \\
\frac{0}{0} \\
\vdots \\
0 \\
0\end{array}$ & 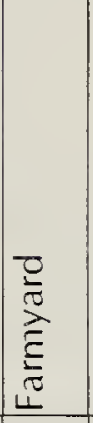 & 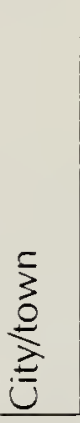 & $\begin{array}{l}\frac{1}{0} \\
3 \\
\frac{0}{0} \\
0 \\
0\end{array}$ & $\frac{\bar{\Phi}}{\overline{0}}$ \\
\hline SNOWDEN & 99 & 3 & 4 & 2.4 & 15 & & & $|6-25|$ & & $26-50 \mid$ & & & & $6-25$ & $6-25$ & & \\
\hline SOMME & 80 & 3 & 5 & 2 & 3 & & $26-50$ & & & & & & $6-25$ & 6-25 $\mid$ & $6-25$ & & \\
\hline SPALDING & 80 & 4 & 4. & 2 & 2 & & $26-50$ & & $6-25$ & $6-25$ & & & & $6-25 \mid$ & & & \\
\hline SPINNEY HILL & 200 & 6 & 1) & .5 & & & & $26-50$ & & $26-50$ & & & & & & & \\
\hline SPRING VALLEY & 66 & 2.5 & 6 & 2 & 1 & & & & & & $6-25$ & & $51-75$ & $26-50$ & & & \\
\hline *SQUAW RAPIDS & 125 & 12 & 18 & 10 & & $6-25$ & $26-50$ & $26-50$ & & & & & & & & & \\
\hline SWIFT CURRENT & 90 & 3 & 3 & 2 & & & & & & & & & & & & & \\
\hline TISDALE & 90 & 2 & & & 4 & & $6-25$ & & $26-50$ & $26-50$ & & & $26-50$ & & & & \\
\hline $\begin{array}{l}\text { TURTLE LAKE } \\
{ }^{*} \text { WEYBURN }\end{array}$ & & & & & 6 & & & & & & & & & & & & \\
\hline "WEYBURN & 356 & 10.5 & 17.5 & 4.75 & 1.5 & $6-25$ & $26-50$ & $6-25$ & $26-50$ & $6-25$ & $6-25$ & & $26-50$ & $6-25$ & $6-25$ & & \\
\hline WHITE BEAR & 14 & \begin{tabular}{|l|}
3 \\
-
\end{tabular} & 17 & 7 & & & & & & & & & & & & & 75 \\
\hline WHITE BEAR LAKE & 129 & 4.5 & 3 & 1 & & & & $26-50 \mid$ & & $26-50$ & & & $26-50$ & & $6-25$ & & \\
\hline WHITEBEECH & 3 & .5 & 2 & 1 & 2 & & & $26-50 \mid$ & $26-50 \mid$ & & & & $6-25 \mid$ & & & & \\
\hline $\begin{array}{l}\text { WHITEWOOD } \\
\text { YORKTON }\end{array}$ & 53 & .75 & 1.6 & .75 & 1 & & & $6-25$ & & $26-50$ & & & & & $6-25$ & & \\
\hline
\end{tabular}

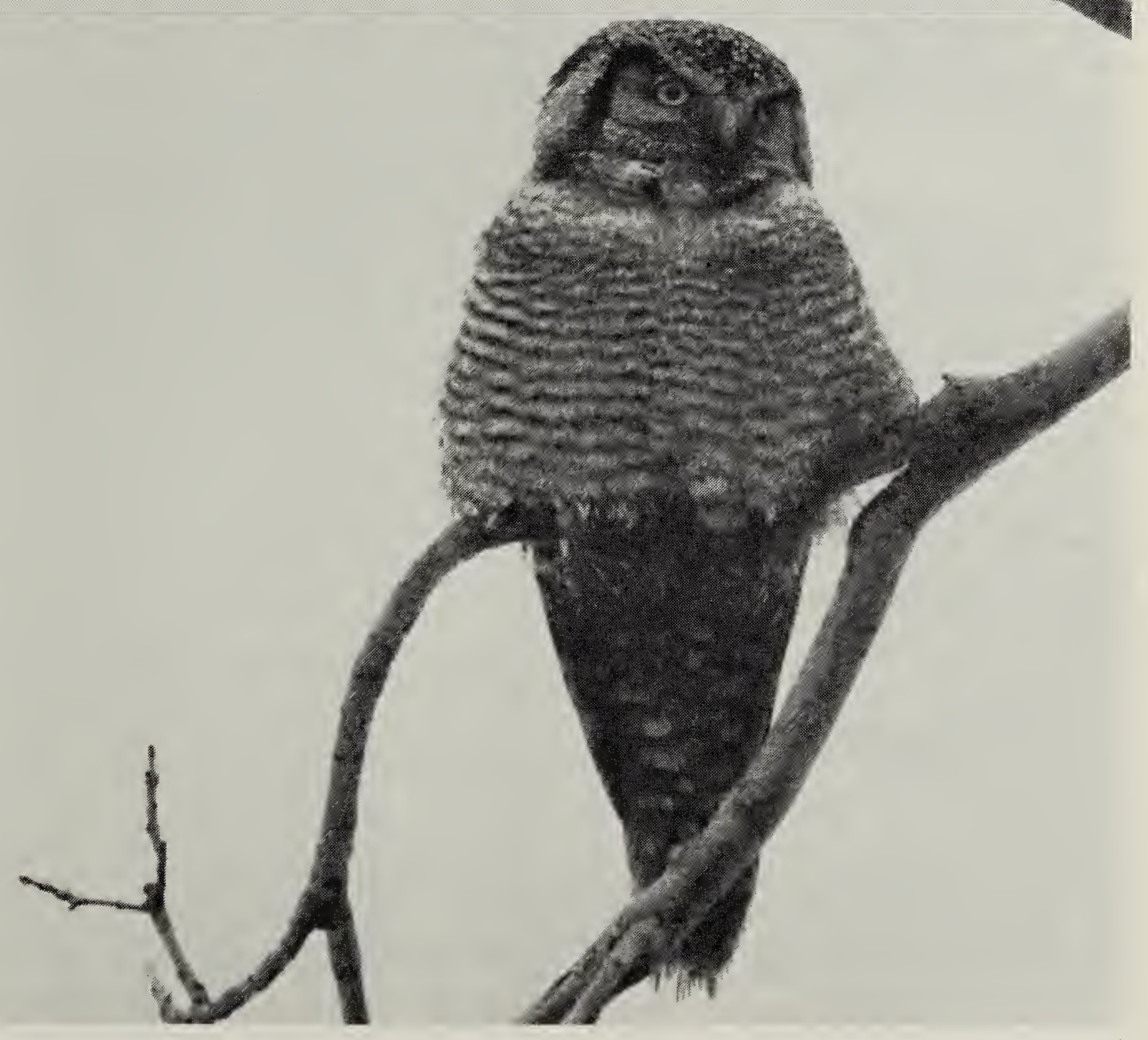


Table 3-1. SPECIES RECORDED FROM MORE THAN FIVE LOCALITIES ( $+=$ a species seen during the count period but not on count day).

\section{SPECIES}

CANADA GOOSE

\section{MALLARD}

COMMON GOLDENEYE

BALD EAGLE

NORTHERN GOSHAWK

GOLDEN EAGLE

MERLIN

PRAIRIE FALCON

GRAY PARTRIDGE

RING-NECKED PHEASANT

RUFFED GROUSE

SHARP-TAILED GROUSE

ROCK DOVE

GREAT HORNED OWL

SNOWY OWL

SHORT-EARED OWL

DOWNY WOODPECKER

HAIRY WOODPECKER

NORTHERN FLICKER

PILEATED WOODPECKER

HORNED LARK

GRAY IAY

BLUE JAY

BLACK-BILLED MAGPIE

COMMON RAVEN

BLACK-CAPPED CHICKADEE

BOREAL CHICKADEE

RED-BREASTED NUTHATCH

WHITE-BREASTED NUTHATCH

BROWN CREEPER

AMERICAN ROBIN

BOHEMIAN WAXWING

CEDAR WAXWING

NORTHERN SHRIKE

EUROPEAN STARLING

AMERICAN TREE SPARROW

DARK-EYED IUNCO

SNOW BUNTING

PINE GROSBEAK

WHITE-WINGED CROSSBILL

COMMON REDPOLL

HOARY REDPOLL

PINE SISKIN

EVENING GROSBEAK

HOUSE SPARROW

NO. SPECIES COUNT DAY

NO. SPECIES COUNT PERIOD

NO. SPECIES TABLE $4 \& 5$

NO. INDIV. TABLES $4 \& 5$

NO. INDIV. COUNT DAY
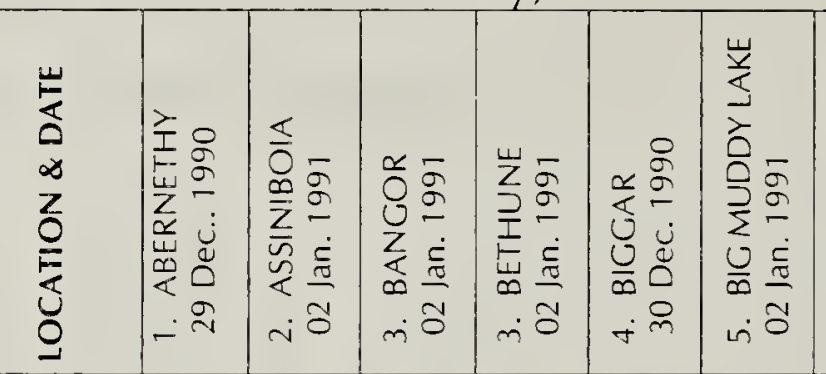
Table 3-2. SPECIES RECORDED FROM MORE THAN FIVE LOCALITIES (+ = a species seen during the count period but not on count day).

\begin{tabular}{|c|c|c|c|c|c|c|c|c|c|c|}
\hline 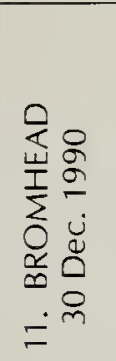 & 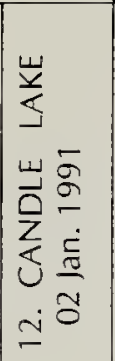 & 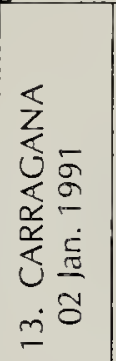 & 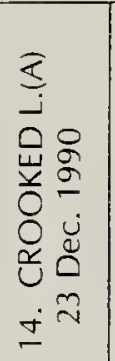 & 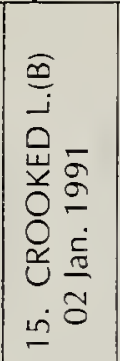 & 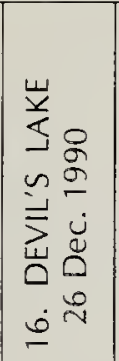 & 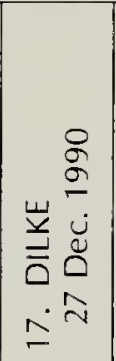 & 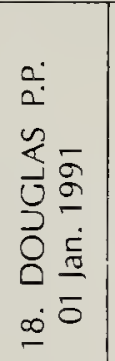 & 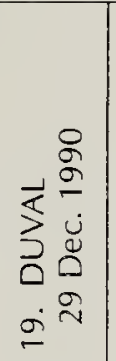 & 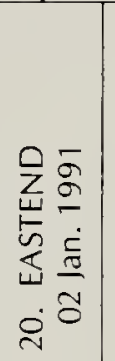 & 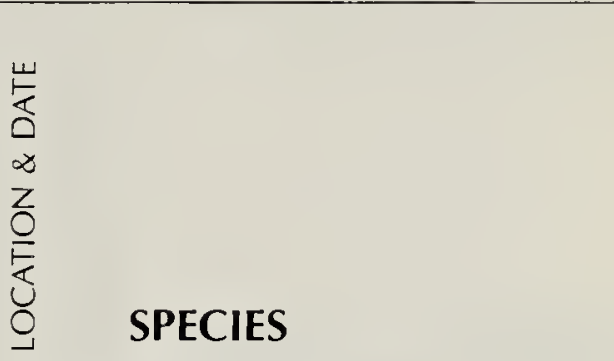 \\
\hline & & & & & & & & & & \multirow{5}{*}{$\begin{array}{l}\text { CANADA GOOSE } \\
\text { MALLARD } \\
\text { COMMON GOLDENEYE } \\
\text { BALD EAGLE } \\
\text { NORTHERN GOSHAWK }\end{array}$} \\
\hline & & & & & & & 4 & & & \\
\hline & & & 2 & & & & 5 & & & \\
\hline & & & & & & & 2 & & 1 & \\
\hline & & & & & & & & & & \\
\hline 2 & & & & & & 1 & 1 & & & \multirow{3}{*}{$\begin{array}{l}\text { GOLDEN EAGLE } \\
\text { MERLIN } \\
\text { PRAIRIE FALCON }\end{array}$} \\
\hline & & & & 1 & & & & & & \\
\hline & & & & & & & & & & \\
\hline 13 & & & 11 & & & + & 9 & 12 & 11 & \multirow{2}{*}{$\begin{array}{l}\text { GRAY PARTRIDGE } \\
\text { RING-NECKED PHEASANT }\end{array}$} \\
\hline 6 & & & & & & & & & 6 & \\
\hline & 5 & 2 & & & & & & & & \multirow{5}{*}{$\begin{array}{l}\text { RUFFED GROUSE } \\
\text { SHARP-TAILED GROUSE } \\
\text { ROCK DOVE } \\
\text { GREAT HORNED OWL } \\
\text { SNOWY OWL }\end{array}$} \\
\hline 12 & 14 & & 22 & & & & 12 & 7 & & \\
\hline 2 & & & & & & 1 & 4 & & & \\
\hline 4 & & & & & & + & 1 & 2 & & \\
\hline 8 & & & & & & 1 & 2 & 2 & 1 & \\
\hline 1 & & & & & & & & & & \multirow{5}{*}{$\begin{array}{l}\text { SHORT-EARED OWL } \\
\text { DOWNY WOODPECKER } \\
\text { HAIRY WOODPECKER } \\
\text { NORTHERN FLICKER } \\
\text { PILEATED WOODPECKER }\end{array}$} \\
\hline & 4 & 1 & 5 & 2 & 2 & 1 & 1 & 2 & & \\
\hline & 1 & 1 & 3 & 3 & 2 & & & & & \\
\hline & & & & & & & & & & \\
\hline & & & & & & & & & & \\
\hline 164 & & & & & & 15 & & 7 & 150 & \multirow{5}{*}{$\begin{array}{l}\text { HORNED LARK } \\
\text { GRAY JAY } \\
\text { BLUE JAY } \\
\text { BLACK-BILLED MAGPIE } \\
\text { COMMON RAVEN }\end{array}$} \\
\hline & 9 & 1 & & & & & & & & \\
\hline & 5 & 1 & 2 & & 5 & & & & & \\
\hline 4 & 31 & 3 & 18 & 3 & 3 & 23 & 6 & 16 & & \\
\hline & 131 & 3 & & 2 & & & & & & \\
\hline & 126 & 4 & 48 & 28 & 11 & & 6 & 9 & & \multirow{5}{*}{$\begin{array}{l}\text { BLACK-CAPPED CHICKADEE } \\
\text { BOREAL CHICKADEE } \\
\text { RED-BREASTED NUTHATCH } \\
\text { WHITE-BREASTED NUTHATCH } \\
\text { BROWN CREEPER }\end{array}$} \\
\hline & $\begin{array}{r}30 \\
\end{array}$ & & & & & & & & & \\
\hline & 6 & & & & & & & & & \\
\hline & & 1 & 2 & 3 & & & & + & & \\
\hline & & & & & & & & & & \\
\hline & & & & & & & & & & \multirow{5}{*}{$\begin{array}{l}\text { AMERICAN ROBIN } \\
\text { BOHEMIAN WAXWING } \\
\text { CEDAR WAXWING } \\
\text { NORTHERN SHRIKE } \\
\text { EUROPEAN STARLING }\end{array}$} \\
\hline & & & & & 11 & & & & & \\
\hline & & & & & & & 19 & & & \\
\hline & & & & & & & & & & \\
\hline & & & & & & & & & & \\
\hline & & & & & & & 4 & & & \multirow{2}{*}{$\begin{array}{l}\text { AMERICAN TREE SPARROW } \\
\text { DARK-EYED JUNCO }\end{array}$} \\
\hline & & & & & & & & & & \\
\hline 2476 & & 50 & & 5 & 30 & + & 15 & 97 & & \multirow{3}{*}{$\begin{array}{l}\text { SNOW BUNTING } \\
\text { PINE GROSBEAK } \\
\text { WHITE-WINGED CROSSBILL }\end{array}$} \\
\hline & 9 & 14 & 4 & & & & & & & \\
\hline & 33 & & & & & & & & & \\
\hline & 3 & 40 & 3. & & & & & + & & \multirow{5}{*}{$\begin{array}{l}\text { COMMON REDPOLL } \\
\text { HOARY REDPOLL } \\
\text { PINE SISKIN } \\
\text { EVENING GROSBEAK } \\
\text { HOUSE SPARROW }\end{array}$} \\
\hline & & & & & & & 3 & & & \\
\hline & & & & & & & & & & \\
\hline & 27 & 4 & & & & & & & & \\
\hline 18 & & & 30 & 40 & 2 & 63 & & 97 & & \\
\hline 13 & 15 & 13 & 12 & 9 & 8 & 7 & 16 & 10 & 5 & \multirow{2}{*}{$\begin{array}{l}\text { NO. SPECIES COUNT DAY } \\
\text { NO. SPECIES COUNT PERIOD }\end{array}$} \\
\hline 13 & 15 & 13 & 12 & 10 & 8 & 10 & 16 & 13 & 5 & \\
\hline 1 & 0 & 0 & 0 & 1 & 0 & 0 & 0 & 1 & 0 & NO. SPECIES TABLE 4 \& 5 \\
\hline 5445 & 0 & 0 & 0 & 0 & 0 & 0 & 0 & 0 & 0 & \multirow{2}{*}{$\begin{array}{l}\text { NO. INDIV. TABLES } 4 \& 5 \\
\text { NO. INDIV. COUNT DAY }\end{array}$} \\
\hline 8155 & 434 & 125 & 150 & 87 & 66 & 105 & $94 \mid$ & 251 & 169 & \\
\hline
\end{tabular}


Table 3-3. SPECIES RECORDED FROM MORE THAN FIVE LOCALITIES $(+=$ a species seen during the count period but not on count day).

\section{SPECIES}

CANADA GOOSE

MALLARD

COMMON GOLDENEYE

BALD EAGLE

NORTHERN GOSHAWK

GOLDEN EAGLE

MERLIN

PRAIRIE FALCON

GRAY PARTRIDGE

RING-NECKED PHEASANT

RUFFED GROUSE

SHARP-TAILED GROUSE

ROCK DOVE

GREAT HORNED OWL

SNOWY OWL

SHORT-EARED OWL

DOWNY WOODPECKER

HAIRY WOODPECKER

NORTHERN FLICKER

PILEATED WOODPECKER

HORNED LARK

GRAY JAY

BLUE JAY

BLACK-BILLED MAGPIE

COMMON RAVEN

BLACK-CAPPED CHICKADEE

BOREAL CHICKADEE

RED-BREASTED NUTHATCH

WHITE-BREASTED NUTHATCH

BROWN CREEPER

AMERICAN ROBIN

BOHEMIAN WAXWING

CEDAR WAXWING

NORTHERN SHRIKE

EUROPEAN STARLING

AMERICAN TREE SPARROW

DARK-EYED JUNCO

SNOW BUNTING

PINE GROSBEAK

WHITE-WINGED CROSSBILL

COMMON REDPOLL

HOARY REDPOLL

PINE SISKIN

EVENING GROSBEAK

HOUSE SPARROW

NO. SPECIES COUNT DAY

NO. SPECIES COUNT PERIOD

NO. SPECIES TABLE $4 \& 5$

NO. INDIV. TABLES 4 \& 5

NO. INDIV. COUNT DAY
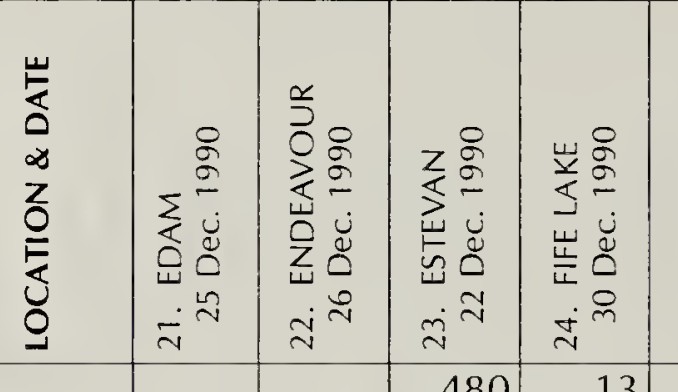

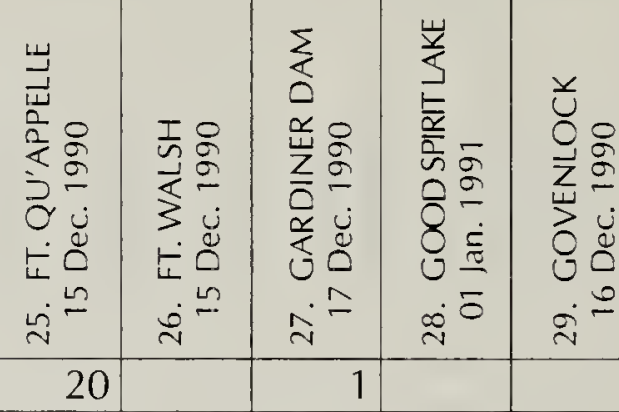

\begin{tabular}{|r|r|r|r|r|r|r|r|r|}
\hline & 480 & 13 & 20 & & 1 & \\
\hline & 99 & 10000 & 229 & 10 & 7100 & \\
\hline 1 & & 2 & 1 & 1 & 1 & 130 & \\
\hline+ & & & & & 15 & 1 \\
\hline
\end{tabular}

\begin{tabular}{|r|r|r|r|r|r|r|r|}
\hline+ & & & 1 & 1 & & + & \\
\hline & & 1 & & 3 & 4 & & 11 \\
\hline & & & & & & & 2 \\
\hline+ & 2 & + & & & & & 3 \\
\hline & 18 & 51 & 9 & 7 & 77 & & 8 \\
\hline & 1 & 2 & 1 & 8 & & & 23 \\
\hline
\end{tabular}

\begin{tabular}{|r|r|r|r|r|r|r|r|r|}
\hline+ & 2 & & & 3 & 1 & & 1 & \\
\hline+ & 6 & 2 & 5 & 7 & 11 & 61 & & 134 \\
\hline & 1 & 35 & & 7 & 7 & 85 & 9 & 37 \\
\hline 1 & 1 & 3 & & 1 & 4 & 5 & & 3 \\
\hline+ & & 2 & & & & 4 & & 10 \\
\hline 5 & & + & 2 & & 1 & & & \\
\hline 3 & 1 & 1 & 1 & 4 & 7 & 1 & 9 & 1 \\
\hline & & & 1 & 1 & 2 & 1 & 8 & \\
\hline & + & & & & & & & \\
\hline & & 53 & 3050 & + & 2 & 37 & & 1551 \\
\hline+ & & & & + & & & & \\
\hline 4 & & & & 25 & & 2 & 3 & \\
\hline 12 & 2 & 15 & & 73 & 140 & 127 & 10 & 22 \\
\hline 6 & 27 & & & + & 2 & 1 & 8 & \\
\hline 18 & 4 & 3 & 1 & 48 & 59 & 9 & 42 & \\
\hline
\end{tabular}

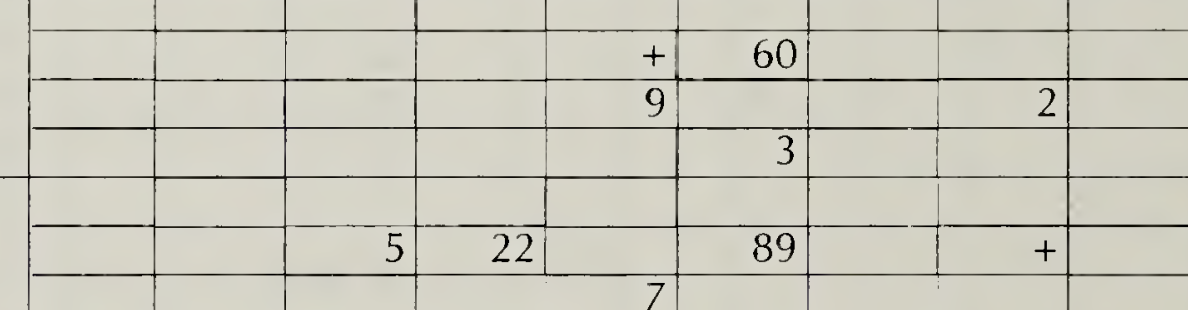

\begin{tabular}{r|r|r|r|r|r|r|r|r}
\hline & & & & & 6 & 2 & & 1 \\
\hline & & + & & 1 & & & & 4 \\
\hline+ & & & & & 26 & & & 3 \\
\hline & 150 & 300 & 1000 & 44 & 67 & 579 & 90 & 432 \\
\hline 4 & 5 & & & + & 2 & & 40 & \\
\hline+ & & & & & 66 & & & \\
\hline & & & & 50 & 95 & 209 & 62 & 75 \\
\hline
\end{tabular}

\begin{tabular}{|r|r|r|r|r|r|r|r|r|}
\hline & & & & & & & & \\
\hline+ & & & & 2 & & & + & \\
\hline 39 & 3 & 10 & 39 & 350 & 193 & 707 & 105 & 347 \\
\hline 10 & 12 & 19 & 15 & 25 & 39 & 28 & 15 & 29 \\
\hline 22 & 13 & 21 & 16 & 30 & 40 & 28 & 19 & 29 \\
\hline 3 & 0 & 1 & 0 & 3 & 11 & 6 & 0 & 8 \\
\hline 0 & 0 & 30 & 0 & 4 & 61 & 715 & 0 & 32 \\
\hline 93 & 206 & 1065 & 14189 & 897 & 958 & 9874 & 396 & 2704 \\
\hline
\end{tabular}


Table 3-4. SPECIES RECORDED FROM MORE THAN FIVE LOCALITIES $(+=$ a species seen during the count period but not on count day).

\begin{tabular}{|c|c|c|c|c|c|c|c|c|c|}
\hline 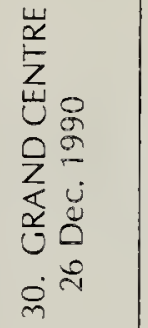 & 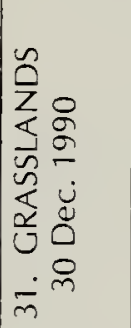 & 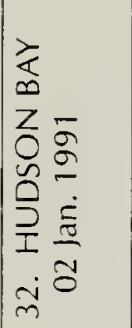 & 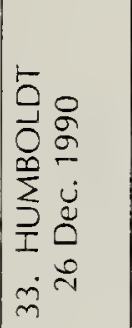 & 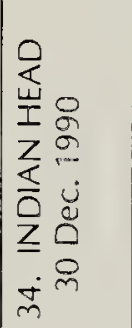 & 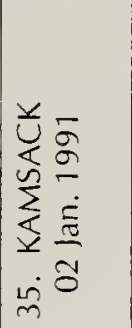 & 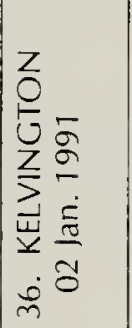 & 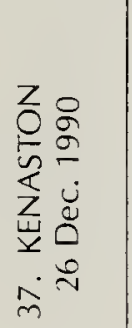 & 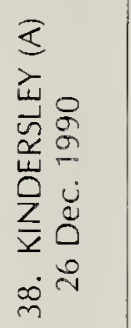 & 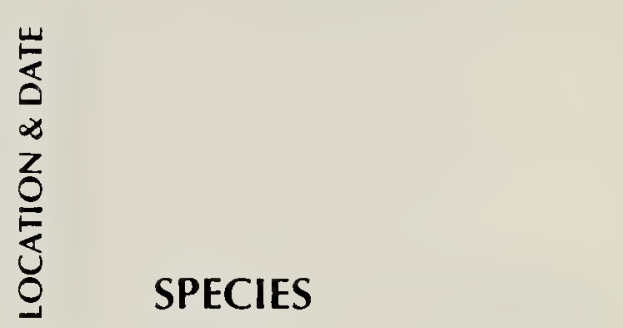 \\
\hline & & & & & & & & & \multirow{5}{*}{$\begin{array}{l}\text { CANADA GOOSE } \\
\text { MALLARD } \\
\text { COMMON GOLDENEYE } \\
\text { BALD EAGLE } \\
\text { NORTHERN GOSHAWK }\end{array}$} \\
\hline & & & & 12 & & & & & \\
\hline & & & & & & & & & \\
\hline & & & & & & & & & \\
\hline & & & & & & & & & \\
\hline 1 & & & & & & & & & \multirow{5}{*}{$\begin{array}{l}\text { GOLDEN EAGLE } \\
\text { MERLIN } \\
\text { PRAIRIE FALCON } \\
\text { GRAY PARTRIDGE } \\
\text { RING-NECKED PHEASANT }\end{array}$} \\
\hline & & & + & & & & & & \\
\hline & & & & & & & & & \\
\hline & 34 & & 8 & 8 & 10 & & 24 & + & \\
\hline & 110 & & & & & & & & \\
\hline+ & & & & 2 & & & & & \multirow{5}{*}{$\begin{array}{l}\text { RUFFED GROUSE } \\
\text { SHARP-TAILED GROUSE } \\
\text { ROCK DOVE } \\
\text { GREAT HORNED OWL } \\
\text { SNOWY OWL }\end{array}$} \\
\hline & 7 & & 27 & 14 & 2 & & 24 & & \\
\hline 6 & & & 80 & 4 & & & 1 & 4 & \\
\hline+ & 2 & & & 5 & 2 & & + & + & \\
\hline & & & + & 2 & & & + & + & \\
\hline & & & & & & & & & \multirow{5}{*}{$\begin{array}{l}\text { SHORT-EARED OWL } \\
\text { DOWNY WOODPECKER } \\
\text { HAIRY WOODPECKER } \\
\text { NORTHERN FLICKER } \\
\text { PILEATED WOODPECKER }\end{array}$} \\
\hline 1 & & & + & 2 & 5 & 2 & & & \\
\hline 4 & & + & & 2 & & 2 & & & \\
\hline & & & & & & & & & \\
\hline 1$)$ & & & & & 3 & & & & \\
\hline & 148 & & & & & & 1 & & \\
\hline 2 & & & & & 9 & & & & GRAY JAY \\
\hline 9 & & 2 & + & 2 & 19 & 4 & & + & BLUE JAY \\
\hline 30 & 111 & & 2 & 30 & 14 & 2 & 5 & 2 & BLACK-BILLED MAGPIE \\
\hline 135 & & + & 2 & 1 & 26 & 2 & & & \multirow{6}{*}{$\begin{array}{l}\text { COMMON RAVEN } \\
\text { BLACK-CAPPED CHICKADEE } \\
\text { BOREAL CHICKADEE } \\
\text { RED-BREASTED NUTHATCH } \\
\text { WHITE-BREASTED NUTHATCH } \\
\text { BROWN CREEPER }\end{array}$} \\
\hline 48 & 2 & 5 & 35 & 66 & 87 & 6 & & & \\
\hline 1 & & & & & 6 & & & & \\
\hline 1 & & & & 9 & 8 & & & & \\
\hline & & 2 & 4 & 2 & 3 & 1 & & & \\
\hline & & & & 1 & & & & & \\
\hline & & & & & & & & & \\
\hline & & & 21 & & 120 & 75 & & + & BOHEMIAN WAXWING \\
\hline & & & & & & & & & CEDAR WAXWING \\
\hline & & & & 1 & & & $+]$ & & NORTHERN SHRIKE \\
\hline & 2 & & & & & & & & EUROPEAN STARLING \\
\hline & & & & & & & & & \multirow{5}{*}{$\begin{array}{l}\text { AMERICAN TREE SPARROW } \\
\text { DARK-EYED UUNCO } \\
\text { SNOW BUNTING } \\
\text { PINE GROSBEAK } \\
\text { WHITE-WINGED CROSSBILL }\end{array}$} \\
\hline+ & & & & 1 & 4 & & & & \\
\hline 5 & 30 & & + & 175 & 75 & 300 & 375 & & \\
\hline 90 & & 12 & 6 & 3 & 92 & & & & \\
\hline 2 & & & & & & & & & \\
\hline 73 & 35 & 5 & 2 & & 38 & & & & \multirow{5}{*}{$\begin{array}{l}\text { COMMON REDPOLL } \\
\text { HOARY REDPOLL } \\
\text { PINE SISKIN } \\
\text { EVENING GROSBEAK } \\
\text { HOUSE SPARROW }\end{array}$} \\
\hline 10 & & & & & & & & & \\
\hline & & & 1 & 2 & 10 & & & & \\
\hline 111 & & 7 & & 4 & 279 & & & & \\
\hline 36 & 110 & & 142 & 157 & 170 & 6 & 15 & 25 & \\
\hline 19 & 13 & 6 & 12 & 24 & 22 & 10 & 7 & 3 & NO SPECIES COUNT DAY \\
\hline 22 & 13 & 9 & 17 & 26 & 22 & 10 & 10 & 8 & NO. SPECIES COUNT PERIOD \\
\hline 0 & 2 & 1 & 0 & 2 & 1 & 0 & 0 & 0 & NO. SPECIES TABLE $4 \& 5$ \\
\hline 0 & 20 & 0 & 0 & 0 & 8 & 0 & 0 & 0 & NO. INDIV. TABLES $4 \& 5$ \\
\hline 566 & 611 & 33 & 330 & 506 & 990 & 400 & 445 & 31 & NO. INDIV. COUNT DAY \\
\hline
\end{tabular}


Table 3-5. SPECIES RECORDED FROM MORE THAN FIVE LOCALITIES ( $+=$ a species seen during the count period but not on count day).

\section{SPECIES}

CANADA GOOSE MALLARD

COMMON GOLDENEYE

BALD EAGLE

NORTHERN GOSHAWK

GOLDEN EAGLE

MERLIN

PRAIRIE FALCON

GRAY PARTRIDGE

RING-NECKED PHEASANT

RUFFED GROUSE

SHARP-TAILED GROUSE

ROCK DOVE

GREAT HORNED OWL

SNOWY OWL

SHORT-EARED OWL

DOWNY WOODPECKER

HAIRY WOODPECKER

NORTHERN FLICKER

PILEATED WOODPECKER

HORNED LARK

GRAY JAY

BLUE JAY

BLACK-BILLED MAGPIE

COMMON RAVEN

BLACK-CAPPED CHICKADEE

BOREAL CHICKADEE

RED-BREASTED NUTHATCH

WHITE-BREASTED NUTHATCH

BROWN CREEPER

AMERICAN ROBIN

BOHEMIAN WAXWING

CEDAR WAXWING

NORTHERN SHRIKE

EUROPEAN STARLING

AMERICAN TREE SPARROW

DARK-EYED JUNCO

SNOW BUNTING

PINE GROSBEAK

WHITE-WINGED CROSSBILL

COMMON REDPOLL

HOARY REDPOLL

PINE SISKIN

EVENING GROSBEAK

HOUSE SPARROW

NO. SPECIES COUNT DAY

NO. SPECIES COUNT PERIOD

NO. SPECIES TABLE $4 \& 5$

NO. INDIV. TABLES 4 \& 5

NO. INDIV. COUNT DAY

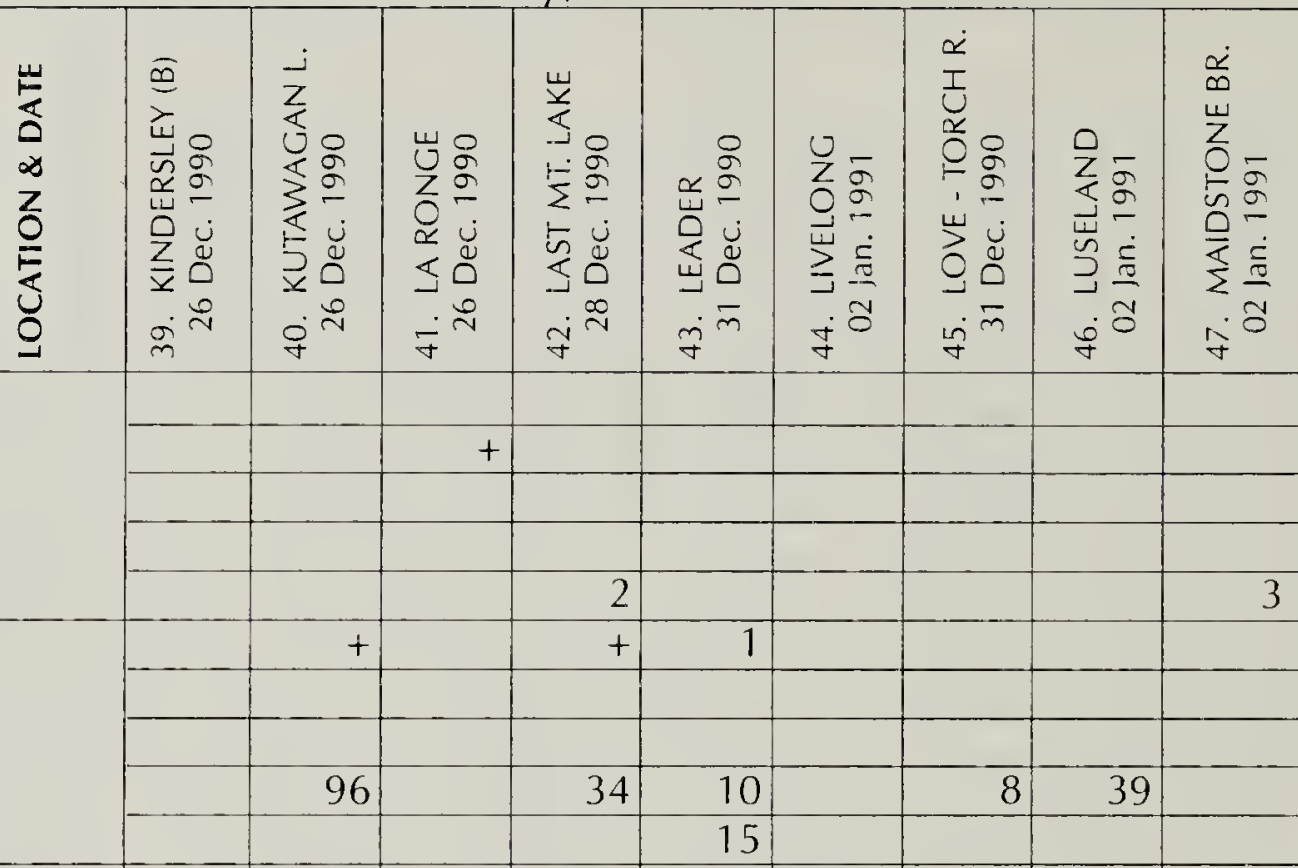

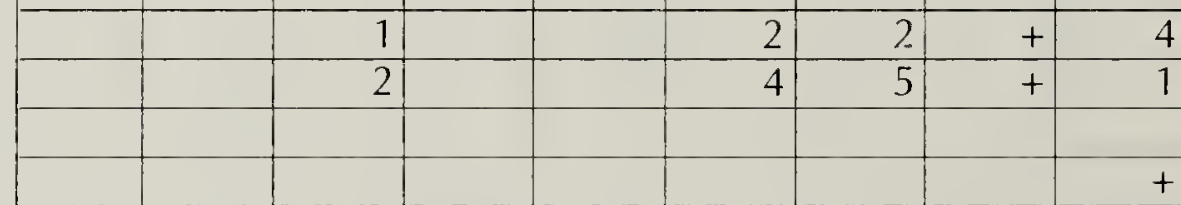

\begin{tabular}{|r|r|r|r|r|r|r|r|r|}
\hline & 1 & & 2 & & & & + & + \\
\hline & & 6 & & & + & 8 & + & \\
\hline 1 & 60 & 4 & & & 20 & 20 & 2 & 25 \\
\hline & & 116 & & & & 22 & & 5 \\
\hline & 1 & 9 & & 2 & 20 & 63 & 12 & 28 \\
\hline & & 5 & & & & & & \\
\hline & & & & & & + & & \\
\hline & & & & & + & 1 & & \\
\hline 35 & & & & & & & & 1 \\
\hline & & & & & & & & \\
\hline & & & & & + & & & \\
\hline & & & & & & & & 1 \\
\hline & & & & + & & & & \\
\hline & 2046 & & 558 & + & 50 & 150 & 337 & 47 \\
\hline & & 24 & & & 30 & 57 & & 11 \\
\hline & & & & & & & & \\
\hline & 50 & & 3 & & + & 33 & 2 & 25 \\
\hline & & & & & & 1 & & \\
\hline & & & & & & 1 & & \\
\hline & & 46 & & & + & 237 & & 3 \\
\hline 18 & 202 & & 75 & 11 & 40 & 16 & 115 & 260 \\
\hline 4 & 11 & 10 & 11 & 8 & 9 & 20 & 11 & 18 \\
\hline 12 & 11 & 13 & 10 & 14 & 22 & 16 & 21 \\
\hline 0 & 0 & 0 & 0 & 0 & 0 & 0 & 0 & 1 \\
\hline 2502 & 215 & 755 & 128 & 188 & 067 & 544 & 488 \\
\hline
\end{tabular}


Table 3-6. SPECIES RECORDED FROM MORE THAN FIVE LOCALITIES (+ = a species seen during the count period but not on count day).

\begin{tabular}{|c|c|c|c|c|c|c|c|c|c|}
\hline 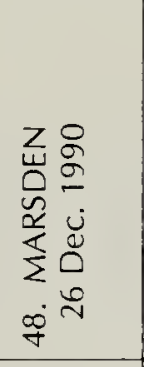 & 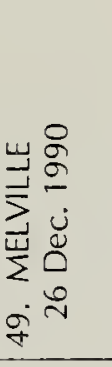 & 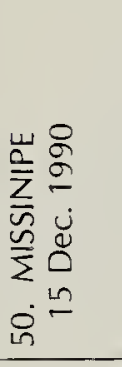 & 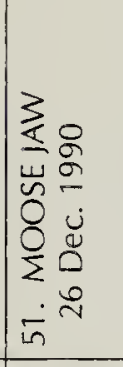 & 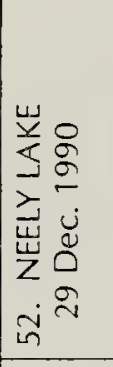 & 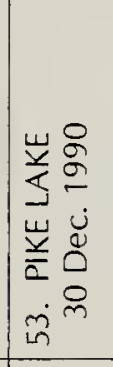 & 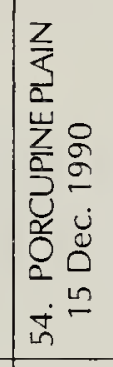 & 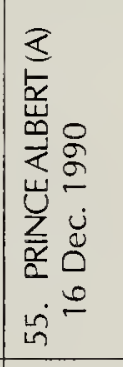 & 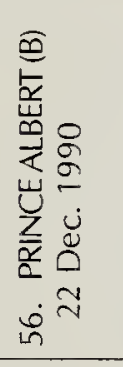 & 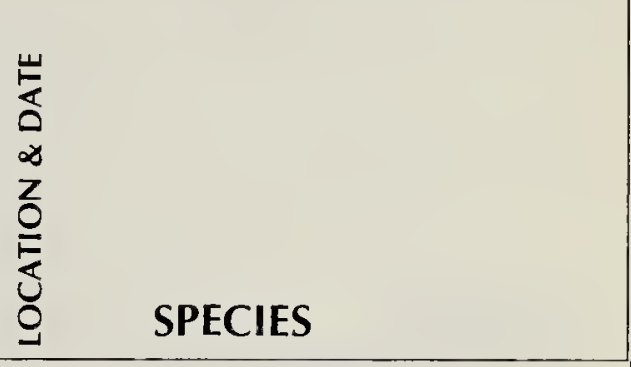 \\
\hline & & & & & & & & & CANADA GOOSE \\
\hline & & & & & & & & & MALLARD \\
\hline & & & & & & & & & COMMON GOLDENEYE \\
\hline & & & & + & & & & & $\begin{array}{l}\text { BALD EAGLE } \\
\text { NORTHERN GOSHAWK }\end{array}$ \\
\hline & & & & & & & & & GOLDEN EAGLE \\
\hline & & & 1 & & & & & & MERLIN \\
\hline & & & & & & & & & PRAIRIE FALCON \\
\hline 24 & & & 15 & & 3. & 1. & & & GRAY PARTRIDGE \\
\hline & & & 2 & & & & & & RING-NECKED PHEASANT \\
\hline$\frac{11}{5}$ & & & & 3 & 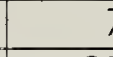 & & & & RUFFED GROUSE \\
\hline 5 & 25 & & & 46 & 10 & & & & SHARP-TAILED GROUSE \\
\hline 6 & 30 & & 155 & & & & & 323 & ROCK DOVE \\
\hline 1 & & & 1 & \pm & & & & + & GREAT HORNED OWL \\
\hline 1 & & & 1 & + & & & & & SNOWY OWL \\
\hline & & & 1 & & & & & & SHORT-EARED OWL \\
\hline 4 & & & 14 & 2 & 17 & & 2 & 2 & DOWNY WOODPECKER \\
\hline 4 & & 1 & 6 & 3 & 12 & & 2 & & HAIRY WOODPECKER \\
\hline 1 & & & 1 & & & & & & NORTHERN FLICKER \\
\hline & & & & 2 & & - & 2 & & PILEATED WOODPECKER \\
\hline 2 & & & 6 & & & & & & HORNED LARK \\
\hline 1 & & 8 & & 8 & & & & & GRAY JAY \\
\hline 13 & & 3 & 1 & s & 15 & 1. & 1 & 7 & BLUE JAY \\
\hline 3 & 1 & & 67 & 19 & $12 !$ & 20 & 1 & 28 & BLACK-BILLED MAGPIE \\
\hline & 3 & & & 19 & & 12 & 4 & 158 & COMMON RAVEN \\
\hline 46 & 3 & 14 & 32 & 14 & 145 & 7. & 8 & 66 & BLACK-CAPPED CHICKADEE \\
\hline & & 15 & & & 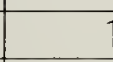 & & & & BOREAL CHICKADEE \\
\hline & & & 7 & & & & & 1 & RED-BREASTED NUTHATCH \\
\hline & & & 3 & 2 & 2 & 2 & 2 & 1 & WHITE-BREASTED NUTHATCH \\
\hline & & & + & & & & & & BROWN CREEPER \\
\hline & & & & & 7 & & & & AMERICAN ROBIN \\
\hline 39 & & & + & & 25 & $4 t$ & 12 & $164 \overline{9}$ & BOHEMIAN WAXWING \\
\hline 1 & & & & & & & & & CEDAR WAXWING \\
\hline & & & + & & 1 & & & & NORTHERN SHRIKE \\
\hline & & & & & & 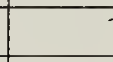 & 1 & & EUROPEAN STARLING \\
\hline & & & & & s & & & & AMERICAN TREE SPARROW \\
\hline & & & 2 & & & & & & DARK-EYED JUNCO \\
\hline 12 & 50 & & & 52 & 169 & - & & 10 & SNOW BUNTING \\
\hline 1 & & 24 & 3 & 12 & 32 & 25 & 4 & 62 & PINE GROSBEAK \\
\hline 4 & & & & & & & & & WHITE-WINGED CROSSBILL \\
\hline & 20 & & 25 & 12 & 376 & 28 & 2 & 3 & COMMON REDPOLL \\
\hline & & & & 2 & 11 & 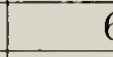 & & + & HOARY REDPOLL \\
\hline & & & & & & & & + & PINE SISKIN \\
\hline 1 & & 20 & & 13 & & 32 & 20 & 131 & EVENING GROSBEAK \\
\hline 1530 & 36 & & 453 & 60 & 647 & 9 & 6 & 184 & HOUSE SPARROW \\
\hline 21 & 8 & 7 & 22 & 18 & 23 & $\overline{2}$ & 14 & 15 & NO. SPECIES COUNT DAY \\
\hline 21 & 8 & 7 & 25 & 27 & 24 & $2 !$ & 14 & 18 & NO. SPECIES COUNT PERIOD \\
\hline 0 & 0 & 0 & 2 & ( & 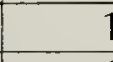 & 2 & 0 & 0 & NO. SPECIES TABLE $4 \& 5$ \\
\hline 0 & 0 & 0 & 5 & ( & 1 & (1) & 0 & 0 & NO. INDIV. TABLES $4 \& 5$ \\
\hline 1710 & 168 & 85 & 801 & 292 & 1739 & 378 & 67 & 2626 & NO. INDIV. COUNT DAY \\
\hline
\end{tabular}


Table 3-7. SPECIES RECORDED FROM MORE THAN FIVE LOCALITIES $(+=$ a species seen during the count period but not on count day).

\section{SPECIES}

\section{CANADA GOOSE}

MALLARD

COMMON GOLDENEYE

BALD EAGLE

NORTHERN GOSHAWK

GOLDEN EAGLE

MERLIN

PRAIRIE FALCON

GRAY PARTRIDGE

RING-NECKED PHEASANT

RUFFED GROUSE

SHARP-TAILED GROUSE

ROCK DOVE

GREAT HORNED OWL

SNOWY OWL

SHORT-EARED OWL

DOWNY WOODPECKER

HAIRY WOODPECKER

NORTHERN FLICKER

PILEATED WOODPECKER

HORNED LARK

GRAY JAY

BLUE JAY

BLACK-BILLED MAGPIE

COMMON RAVEN

BLACK-CAPPED CHICKADEE

BOREAL CHICKADEE

RED-BREASTED NUTHATCH

WHITE-BREASTED NUTHATCH

BROWN CREEPER

AMERICAN ROBIN

BOHEMIAN WAXWING

CEDAR WAXWING

NORTHERN SHRIKE

EUROPEAN STARLING

AMERICAN TREE SPARROW

DARK-EYED JUNCO

SNOW BUNTING

PINE GROSBEAK

WHITE-WINGED CROSSBILL

COMMON REDPOLL

HOARY REDPOLL

PINE SISKIN

EVENING GROSBEAK

HOUSE SPARROW

NO. SPECIES COUNT DAY

NO. SPECIES COUNT PERIOD

NO. SPECIES TABLE $4 \& 5$

NO. INDIV. TABLES $4 \& 5$

NO. INDIV. COUNT DAY

\begin{tabular}{|c|c|c|c|c|c|c|c|c|}
\hline 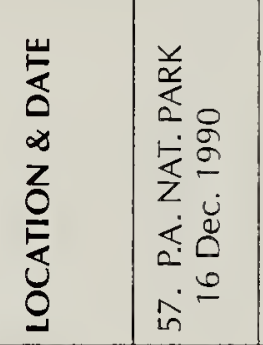 & 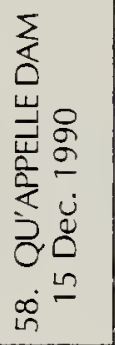 & 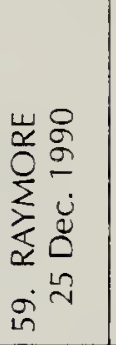 & 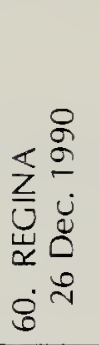 & 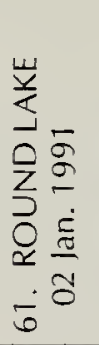 & 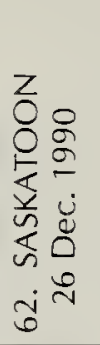 & 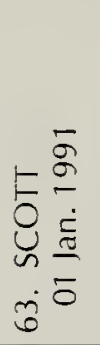 & 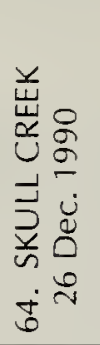 & 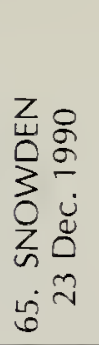 \\
\hline & 317 & & 335 & & & & & \\
\hline & 57 & & 14 & & 1 & & & \\
\hline & 38 & & & & 68 & & & \\
\hline & 6 & + & & & & & + & \\
\hline+ & & 2 & & & & & 1 & 1 \\
\hline & 1 & 1 & & & & & 4 & \\
\hline & & + & 2 & & 1 & & & \\
\hline & & + & & & & & 2 & \\
\hline & & 11 & 96 & 10 & 27 & 31 & + & 8 \\
\hline & & & & & & & 3 & \\
\hline
\end{tabular}

\begin{tabular}{|r|r|r|r|r|r|r|r|r|}
\hline+ & & + & 1 & & 8 & & & 2 \\
\hline & 76 & 47 & 13 & 6 & 54 & & 25 & \\
\hline & 16 & 41 & 672 & 2 & 1547 & 16 & 4 & \\
\hline & 1 & 4 & 10 & 1 & 5 & & 2 & \\
\hline & 3 & 1 & 5 & & 2 & 1 & 2 & \\
\hline
\end{tabular}

\begin{tabular}{|r|r|r|r|r|r|r|r|r|}
\hline & 3 & 1 & 5 & & 2 & 1 & 2 & \\
\hline 12 & & + & 23 & & 1 & & & \\
\hline 13 & 1 & 2 & 6 & 8 & 32 & 2 & 7 & 3 \\
\hline & & & 3 & & 9 & & & \\
\hline 1 & & & & & & & & \\
\hline 9 & 3 & 4 & 2 & & & & 232 & \\
\hline 1 & & + & & & & & & 2 \\
\hline 21 & 31 & 31 & 125 & 4 & 30 & 5 & & 18 \\
\hline 10 & & + & & 28 & 587 & 36 & 13 & 10 \\
\hline 70 & 10 & 9 & 16 & 30 & 285 & 23 & 65 & 48 \\
\hline 19 & & & & & & & & 2 \\
\hline 11 & & + & 10 & & 33 & & & \\
\hline & & & 5 & 10 & & & & 3 \\
\hline & & + & & + & & & & \\
\hline 1 & & & & & 1 & & & \\
\hline & & 35 & & & 2319 & 40 & 285 & \\
\hline
\end{tabular}

\begin{tabular}{|r|r|r|r|r|r|r|r|r|}
\hline & 1 & + & 2 & & 44 & & & \\
\hline & 15 & 6 & 13 & & 45 & & & \\
\hline & & + & & & 2 & & 6 & \\
\hline 3 & 237 & 427 & 937 & 400 & 1076 & 904 & 2500 & 120 \\
\hline 8 & & 7 & & & 19 & & & 8 \\
\hline 46 & & 1 & 5 & & 35 & & & \\
\hline 24 & 7 & 117 & 2 & + & 408 & 40 & 9 & 16 \\
\hline & & 11 & & & 5 & 6 & & \\
\hline & & & 36 & & 1 & & & \\
\hline 21 & & + & & 20 & 1 & & 1 & 160 \\
\hline & 249 & 270 & 1559 & 25 & 4693 & 121 & 84 & 23 \\
\hline 20 & 22 & 21 & 35 & 14 & 40 & 14 & 22 & 18 \\
\hline 22 & 22 & 39 & 36 & 16 & 40 & 14 & 25 & 19 \\
\hline 5 & 4 & 6 & 9 & 0 & 8 & 1 & 4 & 1 \\
\hline 11 & 6 & 1 & 20 & 0 & 72 & 3 & 55 & 0 \\
\hline 280 & 1075 & 1029 & 3924 & 552 & 11450 & 1229 & 3303 & 517 \\
\hline
\end{tabular}


Table 3-8. SPECIES RECORDED FROM MORE THAN FIVE LOCALITIES (+= a species seen during the count period but not on count day).

\begin{tabular}{|c|c|c|c|c|c|c|c|c|c|}
\hline 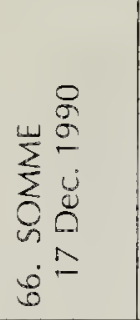 & 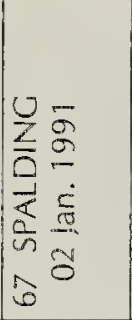 & 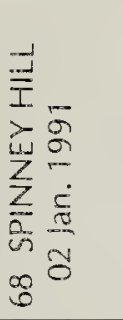 & 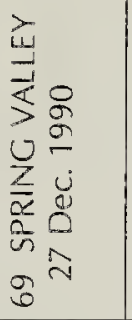 & 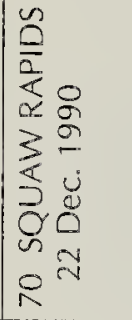 & 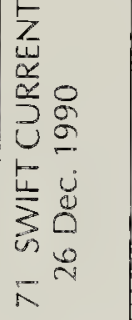 & 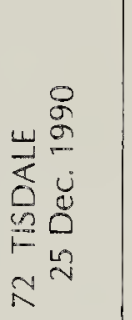 & 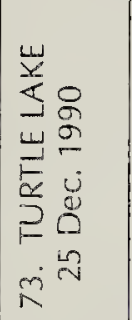 & 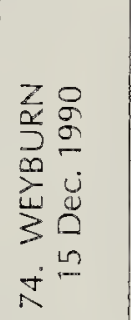 & 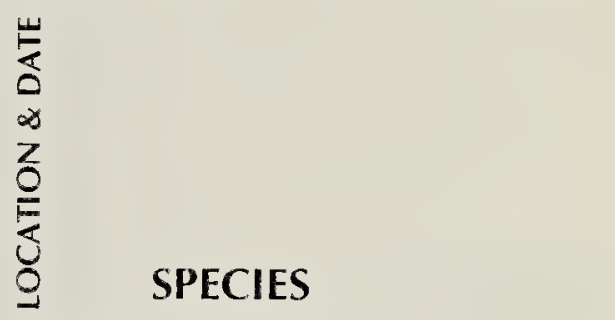 \\
\hline & & & & & & & & + & CANADA GOOSE \\
\hline & & & & 10 & & & & & MALLARD \\
\hline & & & & 275 & & & & & COMMON GOLDENEYE \\
\hline 2 & & & + & 3 & & & & & BALD EAGLE \\
\hline 1 & & & & 10 & & & & & NORTHERN GOSHAWK \\
\hline 1 & & & + & & & & & & GOLDEN EAGLE \\
\hline & & & & & & & & 1 & MERLIN \\
\hline & & & & & & & & 1 & PRAIRIE FALCON \\
\hline 6 & & & 57 & & 4 & 7 & & 44 & GRAY PARTRIDGE \\
\hline & & 1 & & & & & & + & RING-NECKED PHEASANT \\
\hline 2 & 3. & & & & & & 3 & & RUFFED GROUSE \\
\hline & & 18 & 24 & 4 & & & 15 & 44 & SHARP-TAILED GROUSE \\
\hline 5 & 7 & 2 & 15 & & 19 & & & 98 & ROCK DOVE \\
\hline 2 & & 1 & 3 & 1 & & & 3 & 5 & GREAT HORNED OWL \\
\hline & & & + & & & 1 & 2 & 2 & SNOWYOWL \\
\hline & & & 1 & & & & & + & SHORT-EARED OWL \\
\hline 3. & & 2 & + & 2 & & 3 & 6 & 8 & DOWNY WOODPECKER \\
\hline 10 & & 1 & & 3 & & 3. & 7 & 1 & HAIRY WOODPECKER \\
\hline & & & & & 1 & & & & NORTHERN FLICKER \\
\hline 1 & & & & & & 1 & 2 & & PILEATED WOODPECKER \\
\hline & & & 27 & & & & & 172 & HORNED LARK \\
\hline 6 & & & & 1 & & & 12 & & GRAY JAY \\
\hline 16 & & 1 & & 1 & 1 & 6 & $10 \mid$ & & BLUE JAY \\
\hline 22 & 1. & 107 & 5 & 7 & 10 & 4 & 5 & 41 & BLACK-BILLED MAGPIE \\
\hline 81 & 2 & 5 & & 68 & & 4 & 30 & & COMMON RAVEN \\
\hline 16 & 3 & 23 & & 41 & 1 & 8 & 25 & 7 & BLACK-CAPPLD CHICKADEE \\
\hline 7 & & & & 5 & & & 1 & & BOREAL CHICKADEE \\
\hline & & & & 2. & 1. & & ( & 1 & RED-BREASTED NUTHATCH \\
\hline 3 & & & & 1 & 1 & & 7 & 3 & WHITE-BREASTED NUTHATCH \\
\hline & & & & 1 & & & & + & BROWN CREEPER \\
\hline & & & & & 1 & & & 2 & AMERICAN ROBIN \\
\hline 2 & 3 & & 18 & & 19 & 15 & & + & BOHEMIAN WAXWING \\
\hline & & & & & & & & & CEDAR WAXWING \\
\hline+ & & 1 & 1 & & & & & 1 & NORTHERN SHRIKE \\
\hline & & & 10 & & & & 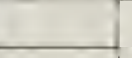 & 6 & EUROPEAN STARLING \\
\hline & & & & & & & & & AMERICAN TREE SPARROW \\
\hline & & & & & 2 & & & 7 & DARK-EYED JUNCO \\
\hline 2 & 1 & 315 & 500 & 73 & & 20 & 25 & 577 & SNOW BUNTING \\
\hline 64 & + & 2. & & 22. & & 20 & 75 & 1 & PINE GROSBEAK \\
\hline & & & & & & & & & WHITE-WINGED CROSSBILL \\
\hline 11 & & 53 & & 16 & & 15 & 15 & 24 & COMMON REDPOLL \\
\hline 2 & & & & 13 & & & & & HOARY REDPOLL \\
\hline & & & & & & & & 6 & PINE SISKIN \\
\hline 43. & & 4 & + & 22 & & 10 & 100 & & EVENING GROSBEAK \\
\hline 82 & 60 & 49 & 360 & 3 & 400 & 20 & & 719 & HOUSE SPARROW \\
\hline 25 & 8 & 16 & 12 & 30 & 13 & 15 & 20. & 25 & NO. SPECIES COUNT DAY \\
\hline 27 & 9 & 16 & 17 & 30 & 13. & 15. & 201 & 32 & NO. SPECIES COUNT PERIOD \\
\hline 2 & 0 & 0 & 0 & 7 & 1. & 0 & 2 & 4 & NO. SPECIES TABLE 4 \& 5 \\
\hline 1 & 0 & 0 & 0 & 18 & 11 & 0 & 3 & 2 & NO. INDIV. TABLES 4 \& 5 \\
\hline 391 & 80 & 585 & 1021 & 602 & 461 & 137 & 346 & 1773 & NO. INDIV. COUNT DAY \\
\hline
\end{tabular}


Table 3-9. SPECIES RECORDED FROM MORE THAN FIVE LOCALITIES ( $+=$ a species seen during the count period but not on count day).

\begin{tabular}{|c|c|c|c|c|c|c|c|c|}
\hline 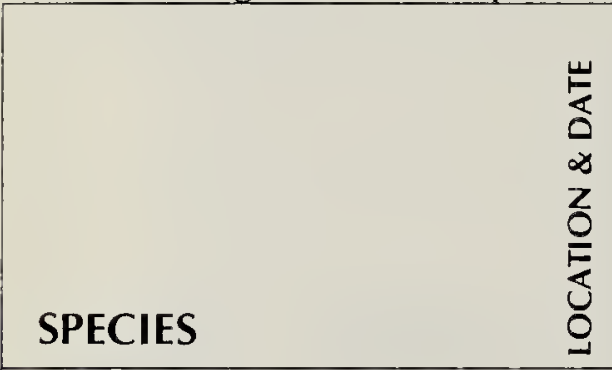 & 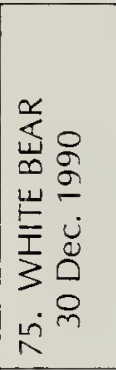 & 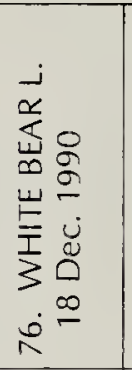 & 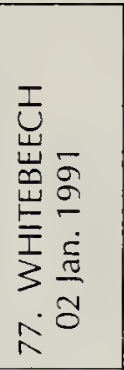 & 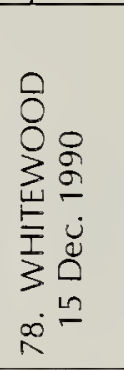 & 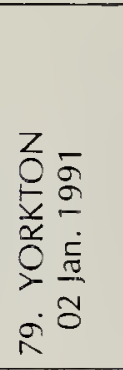 & 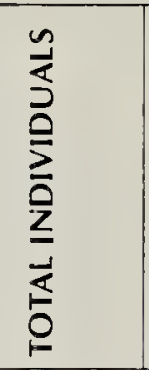 & 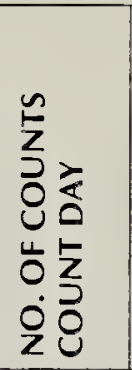 & 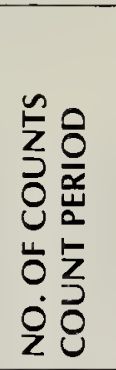 \\
\hline \multirow{5}{*}{$\begin{array}{l}\text { CANADA GOOSE } \\
\text { MALLARD } \\
\text { COMMON GOLDENEYE } \\
\text { BALD EAGLE } \\
\text { NORTHERN GOSHAWK }\end{array}$} & & & & & & 1166 & 6 & 8 \\
\hline & & & & & & 17546 & 12 & 13 \\
\hline & & & & & & 531 & 11 & 11 \\
\hline & & & & & & 33 & 9 & $\overline{12}$ \\
\hline & + & & & + & & 26 & 12 & 18 \\
\hline \multirow{5}{*}{$\begin{array}{l}\text { GOLDEN EAGLE } \\
\text { MERLIN } \\
\text { PRAIRIE FALCON } \\
\text { GRAY PARTRIDGE } \\
\text { RING-NECKED PHEASANT }\end{array}$} & & & & & & 42 & 17 & 22 \\
\hline & & & & & & 10 & 8 & 11 \\
\hline & + & & & & & 10 & 6 & 9 \\
\hline & 74 & & & & 23 & 1170 & 45 & 49 \\
\hline & 6 & 1. & & & 17 & 214 & 18 & 19 \\
\hline \multirow{5}{*}{$\begin{array}{l}\text { RUFFED GROUSE } \\
\text { SHARP-TAILED GROUSE } \\
\text { ROCK DOVE } \\
\text { GREAT HORNED OWL } \\
\text { SNOWY OWL }\end{array}$} & & & 1 & & & 70 & $\overline{24}$ & 30 \\
\hline & 4 & 24 & + & + & 4 & 1149 & 45 & 49 \\
\hline & 360 & 2 & & 40 & 61 & 4072 & 49 & 50 \\
\hline & + & & & & 1 & 99 & 39 & 48 \\
\hline & + & & & 1 & 1 & 59 & 30 & 39 \\
\hline \multirow{5}{*}{$\begin{array}{l}\text { SHORT-EARED OWL } \\
\text { DOWNY WOODPECKER } \\
\text { HAIRY WOODPECKER } \\
\text { NORTHERN FLICKER } \\
\text { PILEATED WOODPECKER }\end{array}$} & 3 & & & & & 42 & 11 & 15 \\
\hline & & & & & 1 & 218 & 52 & 55 \\
\hline & & 9 & 2 & 2 & 2 & 191 & 50 & 52 \\
\hline & & & & & + & 15 & 5 & 6 \\
\hline & & & + & & 1 & 17 & 12 & 15 \\
\hline \multirow{5}{*}{$\begin{array}{l}\text { HORNED LARK } \\
\text { GRAY JAY } \\
\text { BLUE JAY } \\
\text { BLACK-BILLED MAGPIE } \\
\text { COMMON RAVEN }\end{array}$} & + & & & & & 6991 & 24 & 28 \\
\hline & & & & & & 79 & 15 & 19 \\
\hline & & 7 & 7 & & + & 337 & 44 & 49 \\
\hline & 42 & 25 & + & 5 & 43 & 2702 & 73 & 74 \\
\hline & & & 3 & & 3 & 1050 & 40 & 43 \\
\hline \multirow{5}{*}{$\begin{array}{l}\text { BLACK-CAPPED CHICKADEE } \\
\text { BOREAL CHICKADEE } \\
\text { RED-BREASTED NUTHATCH } \\
\text { WHITE-BREASTED NUTHATCH } \\
\text { BROWN CREEPER }\end{array}$} & & 62 & 5 & 10 & 46 & 2127 & 66 & 66 \\
\hline & & & & & & 92 & 11 & 11 \\
\hline & & & & & + & 154 & 15 & 19 \\
\hline & & 6 & + & & 4 & 92 & 30 & 33 \\
\hline & & & & & & 5 & 3 & 7 \\
\hline \multirow{5}{*}{$\begin{array}{l}\text { AMERICAN ROBIN } \\
\text { BOHEMIAN WAXWING } \\
\text { CEDAR WAXWING } \\
\text { NORTHERN SHRIKE } \\
\text { EUROPEAN STARLING }\end{array}$} & & & & & & 7 & 6 & 6 \\
\hline & & & & & & 5174 & 27 & 31 \\
\hline & & & & & 1 & 82 & 7 & 7 \\
\hline & & & & & & 18 & 11 & 18 \\
\hline & & & & & & 105 & 12 & 13 \\
\hline \multirow{2}{*}{$\begin{array}{l}\text { AMERICAN TREE SPARROW } \\
\text { DARK-EYED JUNCO }\end{array}$} & & & & & & 62 & 7 & 9 \\
\hline & & & & & 2 & 62 & 15 & 19 \\
\hline \multirow{4}{*}{$\begin{array}{l}\text { SNOW BUNTING } \\
\text { PINE GROSBEAK } \\
\text { WHITE-WINGED CROSSBILL } \\
\text { COMMON REDPOLL }\end{array}$} & + & & + & + & 252 & 19096 & 57 & 65 \\
\hline & & & 4 & & 2 & 818 & 39 & 41 \\
\hline & & & & & & 192 & 8 & 8 \\
\hline & & 74 & 2 & & 1 & 2294 & 47 & 51 \\
\hline \multirow{2}{*}{$\begin{array}{l}\text { HOARY REDPOLL } \\
\text { PINE SISKIN }\end{array}$} & & & & & & 75 & 14 & 15 \\
\hline & & & & & & 57 & 7 & 8 \\
\hline \multirow{2}{*}{$\begin{array}{l}\text { EVENING GROSBEAK } \\
\text { HOUSE SPARROW }\end{array}$} & & & 40 & & & 1414 & 29 & 35 \\
\hline & 150 & 4 & + & 35 & 389 & 17762 & 67 & 68 \\
\hline NO. SPECIES COUNT DAY & 7 & 10 & 8 & 6 & 19 & & & \\
\hline NO. SPECIES COUNT PERIOD & 14 & 10 & 14 & 9 & 22 & & & \\
\hline NO. SPECIES TABLE $4 \& 5$ & 1 & 0 & 0 & 0 & 0 & & & \\
\hline NO. INDIV. TABLES $4 \& 5$ & 0 & 0 & 0 & 0 & 0 & & & \\
\hline NO. INDIV. COUNT DAY & 639 & 214 & 64 & 93 & 854 & & & \\
\hline
\end{tabular}




\begin{tabular}{|c|c|}
\hline SPECIES & LOCATION AND NUMBER. \\
\hline \multirow{5}{*}{$\begin{array}{l}\text { WESTERN GREBE } \\
\text { TUNDRA SWAN } \\
\text { AMERICAN BLACK DUCK } \\
\text { LESSER SCAUP } \\
\text { HARLEQUIN DUCK }\end{array}$} & Qu'Appelle Dam, 1. \\
\hline & Qu'Appelle Dam, 3. \\
\hline & Gardiner Dam, 1. \\
\hline & Fort Qu'Appelle, 1; Squaw Rapids, 1. \\
\hline & Gardiner Dam, 1. \\
\hline \multirow{5}{*}{$\begin{array}{l}\text { BUFFLEHEAD } \\
\text { COMMON MERGANSER } \\
\text { RED-BREASTED MERGANSER } \\
\text { TURKEY VULTURE } \\
\text { COOPER'S HAWK }\end{array}$} & Qu'Appelle Dam, 1; Squaw Rapids, 1. \\
\hline & Abernethy, 4; Gardiner Dam, 274; Squaw Rapids, 11. \\
\hline & Gardiner Dam, 1; Squaw Rapids, 1. \\
\hline & Edam,.+ \\
\hline & Skull Creek, 1. \\
\hline \multirow{5}{*}{$\begin{array}{l}\text { RED-TAILED HAWK } \\
\text { ROUGH-LEGGED HAWK } \\
\text { AMERICAN KESTREL } \\
\text { PEREGRINE FALCON } \\
\text { GYRFALCON }\end{array}$} & Raymore, +; Squaw Rapids, 1. \\
\hline & Fort Walsh, 2 ; Govenlock, 6 ; Raymore, +. \\
\hline & Crooked Lake $(B),+$; Indian Head, + . \\
\hline & Govenlock, 1. \\
\hline & Biggar, 1; Govenlock, 1; Raymore, +. \\
\hline \multirow{5}{*}{$\begin{array}{l}\text { SPRUCE GROUSE } \\
\text { SAGE GROUSE } \\
\text { WILD TURKEY } \\
\text { KILLDEER } \\
\text { GLAUCOUS GULL }\end{array}$} & Prince Albert National Park, 1; Turtle Lake, 2. \\
\hline & Govenlock, 8; Grasslands National Park, 19. \\
\hline & Fort Qu'Appelle, 2; Fort Walsh, 4; Kamsack, 8. \\
\hline & Govenlock, 1. \\
\hline & Gardiner Dam, 4. \\
\hline \multirow{5}{*}{$\begin{array}{l}\text { NORTHERN HAWK OWL } \\
\text { BARRED OWL } \\
\text { GREAT GRAY OWL } \\
\text { NORTHERN SAW-WHET OWL } \\
\text { THREE-TOED WOODPECKER }\end{array}$} & Abernethy, 1; Fort Qu'Appelle, 1; Raymore, 1; Regina, 1; Squaw Rapids, 1 \\
\hline & Duval, $+;$ Somme, + . \\
\hline & Hudson Bay, +; Prince Albert Nat. Park, 3; Snowden, +; Turtle Lake, 1. \\
\hline & Porcupine Plain, + ; Weyburn,.+ \\
\hline & Fort Walsh, 1; Prince Albert National Park, 1; Somme, 1. \\
\hline \multirow{4}{*}{$\begin{array}{l}\text { BLACK-BACKED WOODPECKER } \\
\text { AMERICAN CROW } \\
\text { GOLDEN-CROWNED KINGLET }\end{array}$} & Squaw Rapids, 1. \\
\hline & Indian Head, 1; Regina, 1; Saskatoon, 4. \\
\hline & $\begin{array}{l}\text { Fort Walsh, 11; Gardiner Dam, 4; Prince Albert National Park, 5; } \\
\text { Raymore, +; Saskatoon, } 4 \text {. }\end{array}$ \\
\hline & Fort Walsh, 1 ; Indian Head, + . \\
\hline \multirow{5}{*}{$\begin{array}{l}\text { VARIED THRUSH } \\
\text { FOX SPARROW } \\
\text { SONG SPARROW } \\
\text { WHITE-THROATED SPARROW } \\
\text { HARRIS' SPARROW } \\
\end{array}$} & Saskatoon, 1. \\
\hline & Raymore,.+ \\
\hline & Fort Wash, 2. \\
\hline & Broadview, +; Porcupine Plain, 1; Regina, 4; Saskatoon, 1. \\
\hline & Fort Walsh, 3; Govenlock, 2; Skull Creek, 2; Weyburn, +. \\
\hline \multirow{5}{*}{$\begin{array}{l}\text { LAPLAND LONGSPUR } \\
\text { RED-WINGED BLACKBIRD } \\
\text { RUSTY BLACKBIRD } \\
\text { BREWER'S BLACKBIRD } \\
\text { COMMON GRACKLE }\end{array}$} & Big Muddy L., 70; Bromhead, 5445; Estevan, 30; Govenlock, 11; Regina, 1 \\
\hline & Fort Walsh, $1 ;$ Weyburn, 1. \\
\hline & Edam, +; Fort Walsh, 24; Govenlock, 2; Regina, 1; Snowden, +. \\
\hline & Moose Jaw, 4; Swift Current, 1; Weyburn, 1. \\
\hline & Moose Jaw, 1; Regina, +; Saskatoon, 1. \\
\hline \multirow{5}{*}{$\begin{array}{l}\text { ROSY FINCH } \\
\text { ROSY FINCH (Gray-crowned) } \\
\text { ROSY FINCH (Black) } \\
\text { PURPLE FINCH } \\
\text { RED CROSSBILL }\end{array}$} & Skull Creek, 52. \\
\hline & Saskatoon, 1 ; White Bear, +. \\
\hline & Big Muddy Lake, 1. \\
\hline & Regina, 1. \\
\hline & Fort Walsh, 9; Regina, 10; Saskatoon, 14; Scott, 3. \\
\hline
\end{tabular}

Table 5. BIRDS NOT IDENTIFIED TO SPECIES

\section{SPECIES}

DUCK species

EAGLE species ACCIPITER species HAWK species FALCON species WOODPECKER species BLACKBIRD species REDPOLL species

\section{LOCATION AND NUMBER}

Gardiner Dam, 400.

Fort Walsh, 1; Grasslands National Park, 1.

Qu'Appelle Dam, 1; Regina, 1.

Bangor, 1.

Edam, +; Fort Walsh, + .

Fort Walsh, 1; Squaw Rapids, 1.

Fort Walsh, 1.

Gardiner Dam, 30; Saskatoon, 45. 
Table 6. SUMMARY OF NEW OR TYING HIGH COUNTS ESTABLISHED DURING THE 1990 COUNTS (tying counts regular type; boldface indicates a new record).

\begin{tabular}{|c|c|c|c|c|}
\hline LOCATION & $\begin{array}{r}1990 \\
\text { COUNT }\end{array}$ & SPECIES & $\begin{array}{l}\text { VIOUS } \\
\text { HIGH }\end{array}$ & LOCATION AND YEAR \\
\hline Qu'Appelle Dam & 1 & Western Grebe & 1 & Gardiner Dam 79,88; Regina 56,59 \\
\hline Gardiner Dam & 1 & American Black Duck & 1 & Regina 73; Gardiner Dam 88 \\
\hline Fife Lake & 10000 & Mallard & 8956 & Gardiner Dam 88 \\
\hline Gardiner Dam & 1 & Harlequin Duck & & NEW \\
\hline Gardiner Dam & 274 & Common Merganser & 164 & Gardiner Dam 89 \\
\hline Skull Creek & 1 & Cooper's Hawk & 1 & 9 records n. to Battleford \\
\hline Govenlock & 1 & Peregrine Falcon & 1 & $\begin{array}{l}17 \text { records } n \text {. to Squaw Rapids- } \\
\text { Carrot River }\end{array}$ \\
\hline Govenlock & 1 & Killdeer & 1 & Govenlock 82 \\
\hline Gardiner Dam & 4 & Glaucous Gull & 4 & Gardiner Dam 87,88 \\
\hline Regina & 23 & Short-eared Owl & 23 & Regina 69 \\
\hline Saskatoon & 32 & Downy Woodpecker & 31 & Saskatoon 88 \\
\hline Saskatoon & 28 & Hairy Woodpecker & 25 & Saskatoon 86 \\
\hline Saskatoon & 9 & Northern Flicker & 9 & Saskatoon \\
\hline Fife Lake & 3050 & Horned Lark & 3043 & Govenlock 83 \\
\hline Saskatoon & 587 & Black-billed Magpie & 452 & Saskatoon 86 \\
\hline Saskatoon & 285 & Black-capped Chickadee & 270 & Saskatoon 86 \\
\hline Saskatoon & 1 & Varied Thrush & 1 & 5 records n. to Saskatoon \\
\hline Fort Walsh & 6 & Northern Shrike & 6 & $\begin{array}{l}\text { Saskatoon } 63 \text {; Pike Lake } 69 \text {; } \\
\text { Fort Walsh } 89\end{array}$ \\
\hline Fort Walsh & 2 & Song Sparrow & 2 & Fort Qu'Appelle 88 \\
\hline Fort Walsh & 3 & Harris'Sparrow & 3 & Govenlock 86 \\
\hline Bromhead & 5445 & Lapland Longspur & 5272 & Big Muddy Lake 88 \\
\hline Big Muddy Lake & 1 & Rosy Finch (Black) & & NEW \\
\hline
\end{tabular}

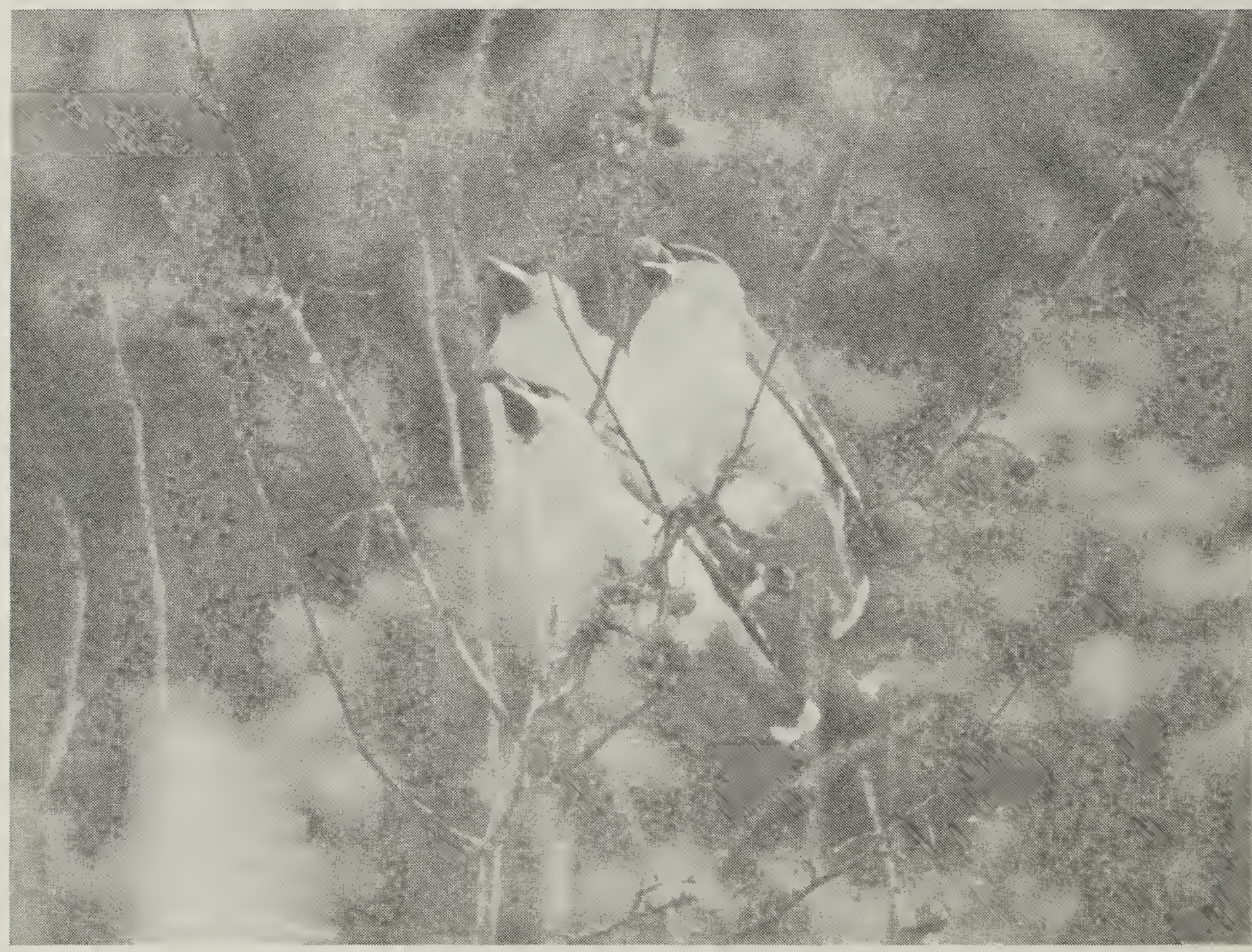

